\title{
Critical growth elliptic problems involving Hardy-Littlewood-Sobolev critical exponent in non-contractible domains
}

https://doi.org/10.1515/anona-2020-0026

Received December 18, 2018; accepted March 8, 2019.

Abstract: The paper is concerned with the existence and multiplicity of positive solutions of the nonhomogeneous Choquard equation over an annular type bounded domain. Precisely, we consider the following equation

$$
-\Delta u=\left(\int_{\Omega} \frac{|u(y)|^{2_{\mu}^{*}}}{|x-y|^{\mu}} d y\right)|u|^{2_{\mu}^{*}-2} u+f \text { in } \Omega, \quad u=0 \text { on } \partial \Omega,
$$

where $\Omega$ is a smooth bounded annular domain in $\mathbb{R}^{N}(N \geq 3), 2_{\mu}^{\star}=\frac{2 N-\mu}{N-2}, f \in L^{\infty}(\Omega)$ and $f \geq 0$. We prove the existence of four positive solutions of the above problem using the Lusternik-Schnirelmann theory and varitaional methods, when the inner hole of the annulus is sufficiently small.

Keywords: Hardy-Littlewood-Sobolev inequality, critical problems, non-contractible domains

MSC: 35A15, 35J60, 35J20

\section{Introduction}

In the pioneering work, Tarantello [31] studied the nonhomogeneous elliptic equation

$$
-\Delta u=|u|^{2^{*}-2} u+f \text { in } \Omega, u=0 \text { on } \partial \Omega,
$$

where $2^{\star}=\frac{2 N}{N-2}$ is the critical Sobolev exponent and $\Omega$ is a bounded domain in $\mathbb{R}^{N}$ with smooth boundary. If $f \in H^{-1}$ then it is shown that there exists at least two solutions of (1.1) by using variational methods. Cao and Zhou [9] proved the existence of two positive solutions of the following nonhomogeneous elliptic equation

$$
-\Delta u=f(x, u(x))+h \text { in } \mathbb{R}^{N}
$$

where $f(x, u)$ is a Carathéodory function with subcritical grotwh at $\infty$. Further, many researchers investigated (1.1) and (1.2) for the existence and multiplicity of solutions. For details, we refer [10,11, 20, 21, 33] and references therein. Recently, Gao and Yang [30] proved the existence of two positive solutions of the nonhomogeneous Choquard equation involving Hardy-Littlewood-Sobolev critical exponent using the splitting Nehari manifold method of Tarantello [31].

Divya Goel, Department of Mathematics, Indian Institute of Technology Delhi, Hauz Khaz, New Delhi-110016, India, E-mail: divyagoel2511@gmail.com

*Corresponding Author: Konijeti Sreenadh, Department of Mathematics, Indian Institute of Technology Delhi, Hauz Khaz, New Delhi-110016, India, E-mail: sreenadh@maths.iitd.ac.in 
The existence, uniqueness, and multiplicity of positive solutions of the nonlocal elliptic equation, precisely the Choquard equation both for mathematical analysis and in perspective of physical models has recently gained significant attention amongst researchers. As an instance, in 1954 Pekar [28] proposed the equation

$$
-\Delta u+u=\left(\frac{1}{|x|} \star|u|^{2}\right) u \text { in } \mathbb{R}^{3}
$$

to study the quantum theory of polaron. Later in 1976, Ph. Choquard [22] examined the steady state of one component plasma approximation in Hartee-Fock theory using (1.3). In [22], Leib proved the existence and uniqueness of the ground state of (1.3). The work of Moroz and Schaftingen enriches the literature of Choquard equations. In [25] authors studied the following Choquard equation

$$
-\Delta u+V u=\left(I_{\alpha} \star F(u)\right) F^{\prime}(u), \quad \text { in } \mathbb{R}^{N},
$$

where $\alpha \in(0, N), N \geq 3, I_{\alpha}$ is the Riesz Potential and $F(u) \in C^{1}(\mathbb{R}, \mathbb{R})$ with sub critical growth. In this work authors established the existence of ground state soloutions of (1.4) and assuming some suitable growth conditions on $F$ and $V$, they studied the properties like constant sign solutions and radial symmetry of the solution. Moreover, authors proved the Pohožaev identity and nonlocal Brezis-Kato type estimate. Interested readers are referred to $[16,24,26,27]$ and references therein for the study of Choquard equation on the unbounded domain.

Concerning the boundary value problems of Choquard equation, Gao and Yang [15] studied the BrezisNirenberg type existence results for the following critical equation

$$
-\Delta u=\lambda h(u)+\left(\int_{\Omega} \frac{|u(y)|^{2_{\mu}^{*}}}{|x-y|^{\mu}} d y\right)|u|^{2_{\mu}^{*}-2} u \text { in } \Omega, \quad u=0 \text { on } \partial \Omega,
$$

where $\lambda>0,0<\mu<N, h(u)=u, \Omega$ is a smooth bounded domain in $\mathbb{R}^{N}$. Later in [14] authors proved the existence and multiplicity of positive solutions for convex and convex-concave type nonlinearities $(h(u)=$ $\left.u^{q}, 0<q<1\right)$ using variational methods.

The geometry of the domain $\Omega$ plays an essential and significant role on the existence and multiplicity of the elliptic boundary value problems. Indeed, in [12], Coron proved the existence of a high energy positive solution of the problem

$$
-\Delta u=|u|^{2^{*}-2} u \text { in } \Omega, \quad u=0 \text { on } \partial \Omega,
$$

where $\Omega$ is a bounded domain in $\mathbb{R}^{N}(N \geq 3)$, precisely an annulus with a small hole. Later in [3], Bahri and Coron, proved that a positive solution always exists as long as the domain has non-trivial homology with $\mathbb{Z}_{2}$-coefficients. In [6], Benci and Cerami studied the following equation

$$
-\varepsilon \Delta u+u=f(u) \text { in } \Omega, \quad u=0 \text { on } \partial \Omega,
$$

where $\varepsilon \in \mathbb{R}^{+}, \Omega$ is a bounded domain in $\mathbb{R}^{N}(N \geq 3)$ and $f: \mathbb{R}_{+} \rightarrow \mathbb{R}$ is a $C^{1,1}$ function. Here authors proved that there exists $\epsilon^{\star}>0$ such that for all $\varepsilon \in\left(0, \epsilon^{\star}\right),(1.6)$ has cat $(\Omega)+1$ solutions under some growth conditions on the function $f$. Since then, the study of existence and multiplicity of solutions of elliptic equations over non-contractible domain has been substantially studied, for instance, $[4,5,13,20,29,32]$ and references therein.

The existence of high energy solution of (1.5) is a much more delicate issue. In this spirit, recently Goel, Rădulescu and Sreenadh [19] studied the Coron problem for Choquard equations. Here authors proved the existence of a positive high energy solution for the problem $\left(P_{f}\right)$ when $f(x) \equiv 0$ and $\Omega$ is a smooth bounded domain in $\mathbb{R}^{N}(N \geq 3)$ satisfying the following condition

(A) There exists constants $0<R_{1}<R_{2}<\infty$ such that

$$
\left\{x \in \mathbb{R}^{N}: R_{1}<|x|<R_{2}\right\} \subset \Omega, \quad\left\{x \in \mathbb{R}^{N}:|x|<R_{1}\right\} \nsubseteq \bar{\Omega} .
$$


In the light of above works, in this article, we study following problem

$$
\left(P_{f}\right)\left\{-\Delta u=\left(\int_{\Omega} \frac{\left|u^{+}(y)\right|^{2_{\mu}^{*}}}{|x-y|^{\mu}} d y\right)\left|u^{+}\right|^{2_{\mu}^{*}-2} u^{+}+f, \text { in } \Omega, u=0 \text { on } \partial \Omega,\right.
$$

where $2_{\mu}^{\star}=\frac{2 N-\mu}{N-2}$, is the critical exponent in the sense of Hardy-Littlewood-Sobolev inequality (2.1) and $f \in \hat{F}$ with $\hat{F}:=\left\{f: f \in L^{\infty}(\Omega), f \geq 0, f \not \equiv 0\right\}$. The domain $\Omega \subset \mathbb{R}^{N}(N \geq 3)$ satisfies the condition $(A)$. Here we prove the existence of four solutions of the problem $\left(P_{f}\right)$. To achieve this, we first seek the help of Nehari manifold associated with $\left(P_{f}\right)$ to prove the existence of the first solution (say $u_{1}$ ). To proceed further, we prove many new estimates on the convolution terms involving the minimizers of best constant $S_{H, L}$ (see Lemma 4.1, 4.3 and 4.4). With the help of these estimates we prove that the minima of the functional over $\mathcal{N}_{f}$ is below the first critical level where the first critical level is

$$
\partial_{f}\left(u_{1}\right)+\frac{N-\mu+2}{2(2 N-\mu)} S_{H, L}^{\frac{2 N-\mu}{N-\mu+2}} .
$$

Here $\mathcal{J}_{f}$ is the energy functional associated to $\left(P_{f}\right)$ (defined in (2.3)). Moreover, $\partial_{f}$ satisfies the Palais-Smale condition below the first critical level. Subsequently, we show the existence of the second and the third solution of $\left(P_{f}\right)$, in $\mathcal{N}_{f}^{-}$(a closed subset of the Nehari manifold) by using a well-known result of Ambrosetti [2](see Lemma 5.2) and assumption $(A)$. To study the existence of the fourth solution, a high energy solution, we prove that the functional $\mathcal{J}_{f}$ satisfies the Palais-Smale condition between the first and the second critical levels, where the second critical level is

$$
\inf _{u \in \mathcal{N}_{f}^{-}} \partial_{f}(u)+\frac{N-\mu+2}{2(2 N-\mu)} S_{H, L}^{\frac{2 N-\mu}{N-\mu+2}} .
$$

To prove the existence of fourth solution, we use the minmax Lemma (See Lemma 6.6). To the best of our knowledge, there is no work on the existence and multiplicity of solutions to Choquard equations $\left(P_{f}\right)$ in non-contractible domains. With this introduction, we state our main result.

Theorem 1.1. Assume $\mu<\min \{4, N\}, f \in L^{\infty}(\Omega)$ and $f \geq 0$ and $\Omega$ be a bounded domain satisfying the conditon (A). Then there exists $e^{\star}>0$ such that $\left(P_{f}\right)$ has at least three positive solutions whenever $0<\|f\|_{H^{-1}}<e^{\star}$. Moreover, if $R_{1}$ is small enough then there exists $e^{\star \star}>0$ such that $\left(P_{f}\right)$ has at least four positive solutions whenever $0<\|f\|_{H^{-1}}<e^{\star \star}$.

The paper is organized as follows: In Section 2, we give the variational framework and preliminary results. In section 3, using the Nehari manifold technique, we prove the existence of the first solution. In section 4, we prove some crucial estimates of the minimizer of $S_{H, L}$ (defined in (2.2)) and analyze the Palais-Smale sequences. In section 5 , we prove the existence of the second and third solution. In section 6 , we prove the existence of the fourth solution.

\section{Variational framework and preliminary results}

We start with the familiar Hardy-Littlewood-Sobolev Inequality which leads to the study of nonlocal Choquard equation using variational methods.

Proposition 2.1. [23](Hardy-Littlewood-Sobolev Inequality) Let $t, r>1$ and $0<\mu<N$ with $1 / t+\mu / N+1 / r=$ 2, $f \in L^{t}\left(\mathbb{R}^{N}\right)$ and $h \in L^{r}\left(\mathbb{R}^{N}\right)$. There exists a sharp constant $C(t, r, \mu, N)$ independent of $f$ and $h$ such that

$$
\int_{\mathbb{R}^{N}} \int_{\mathbb{R}^{N}} \frac{f(x) h(y)}{|x-y|^{\mu}} d x d y \leq C(t, r, \mu, N)\|f\|_{L^{t}\left(\mathbb{R}^{N}\right)}\|h\|_{L^{r}\left(\mathbb{R}^{N}\right)} .
$$

If $t=r=2 N /(2 N-\mu)$, then

$$
C(t, r, \mu, N)=C(N, \mu)=\pi^{\frac{\mu}{2}} \frac{\Gamma\left(\frac{N}{2}-\frac{\mu}{2}\right)}{\Gamma\left(N-\frac{\mu}{2}\right)}\left\{\frac{\Gamma\left(\frac{N}{2}\right)}{\Gamma\left(\frac{\mu}{2}\right)}\right\}^{-1+\frac{\mu}{N}} .
$$


Equality holds in (2.1) if and only if $f \equiv($ constant $) h$ and

$$
h(x)=A\left(y^{2}+|x-a|^{2}\right)^{(2 N-\mu) / 2},
$$

for some $A \in \mathbb{C}, 0 \neq y \in \mathbb{R}$ and $a \in \mathbb{R}^{N}$.

The best constant for the embedding $D^{1,2}\left(\mathbb{R}^{N}\right)$ into $L^{2^{\star}}\left(\mathbb{R}^{N}\right)\left(\right.$ where $\left.2^{\star}=\frac{2 N}{N-2}\right)$ is defined as

$$
S=\inf _{u \in D^{1,2}\left(\mathbb{R}^{N}\right) \backslash\{0\}}\left\{\int_{\mathbb{R}^{N}}|\nabla u|^{2} d x: \int_{\mathbb{R}^{N}}|u|^{2^{\star}} d x=1\right\} .
$$

Consequently, we define

$$
S_{H, L}=\inf _{u \in D^{1,2}\left(\mathbb{R}^{N}\right) \backslash\{0\}}\left\{\int_{\mathbb{R}^{N}}|\nabla u|^{2} d x: \int_{\mathbb{R}^{N}} \int_{\mathbb{R}^{N}} \frac{|u(x)|^{2_{\mu}^{*}}|u(y)|^{2_{\mu}^{*}}}{|x-y|^{\mu}} d x d y=1\right\} .
$$

Lemma 2.2. [15] The constant $S_{H, L}$ defined in (2.2) is achieved if and only if

$$
u=C\left(\frac{b}{b^{2}+|x-a|^{2}}\right)^{\frac{N-2}{2}}
$$

where $C>0$ is a fixed constant, $a \in \mathbb{R}^{N}$ and $b \in(0, \infty)$ are parameters. Moreover,

$$
S=S_{H, L}(C(N, \mu))^{\frac{N-2}{2 N-\mu}} .
$$

Lemma 2.3. [15] For $N \geq 3$ and $0<\mu<N$. Then

$$
\|\cdot\|_{N L}:=\left(\int_{\mathbb{R}^{N}} \int_{\mathbb{R}^{N}} \frac{|\cdot|^{2_{\mu}^{*}}|\cdot|^{2_{\mu}^{*}}}{|x-y|^{\mu}} d x d y\right)^{\frac{1}{2.2_{\mu}^{*}}}
$$

defines a norm on $L^{2^{*}}\left(\mathbb{R}^{N}\right)$.

The energy functional $\mathcal{J}_{f}: H_{0}^{1}(\Omega) \rightarrow \mathbb{R}$ associated with the problem $\left(P_{f}\right)$ is

$$
\partial_{f}(u)=\frac{1}{2} \int_{\Omega}|\nabla u|^{2} d x-\frac{1}{2.2_{\mu}^{\star}} \int_{\Omega} \int_{\Omega} \frac{\left|u^{+}(x)\right|^{2_{\mu}^{*}}\left|u^{+}(y)\right|^{2_{\mu}^{*}}}{|x-y|^{\mu}} d x d y-\int_{\Omega} f u d x,
$$

where $u^{+}=\max (u, 0)$. By using Hardy-Littlewood-Sobolev inequality (2.1), we have

$$
\left(\int_{\Omega} \int_{\Omega} \frac{\left|u^{+}(x)\right|^{2_{\mu}^{*}}\left|u^{+}(y)\right|^{2_{\mu}^{*}}}{|x-y|^{\mu}} d x d y\right)^{\frac{1}{2_{\mu}^{\star}}} \leq C(N, \mu)^{\frac{2 N-\mu}{N-2}}|u|_{2^{\star}}^{2}
$$

It is not difficult to show that the functional $\partial_{f} \in C^{1}\left(H_{0}^{1}(\Omega), \mathbb{R}\right)$ and moreover, if $\mu<\min \{4, N\}$ then $\partial_{f} \in$ $C^{2}\left(H_{0}^{1}(\Omega), \mathbb{R}\right)$.

Definition 2.4. A function $u \in H_{0}^{1}(\Omega)$ is called a weak solution of the problem $\left(P_{f}\right)$ if for all $v \in H_{0}^{1}(\Omega)$ the following holds

$$
\int_{\Omega} \nabla u \cdot \nabla v d x-\int_{\Omega} \int_{\Omega} \frac{\left|u^{+}(x)\right|^{2_{\mu}^{*}}\left|u^{+}(y)\right|^{2_{\mu}^{*}-1} v(y)}{|x-y|^{\mu}} d x d y-\int_{\Omega} f v d x=0 .
$$

Definition 2.5. For $c \in \mathbb{R},\left\{u_{n}\right\}$ is a $(P S)_{c}$ sequence in $H_{0}^{1}(\Omega)$ for $\mathcal{J}_{f}$ if $\mathcal{J}_{f}=c+o(1)$ and $\mathcal{J}_{f}^{\prime}\left(u_{n}\right)=o(1)$ strongly in $H^{-1}$ as $n \rightarrow \infty$. We say $\mathcal{J}_{f}$ satisfies the $(P S)_{c}$ condition in $H_{0}^{1}(\Omega)$ if every $(P S)_{c}$ sequence in $H_{0}^{1}(\Omega)$ has a convergent subsequence. 
Since $\mathcal{J}_{f}$ is not bounded below on $H_{0}^{1}(\Omega)$, it is worth to consider the Nehari manifold

$$
\mathcal{N}_{f}:=\left\{u \in H_{0}^{1}(\Omega) \backslash\{0\} \mid u^{+} \not \equiv 0 \text { and }\left\langle\partial_{f}^{\prime}(u), u\right\rangle=0\right\},
$$

where $\langle$,$\rangle denotes the usual duality. We define$

$$
\Upsilon_{f}=\inf _{u \in \mathcal{N}_{f}} \mathcal{J}_{f}(u)
$$

Note that when $f(x) \equiv 0, \Upsilon_{0}(\Omega)$ is independednt of $\Omega$ and $\Upsilon_{0}(\Omega):=\Upsilon_{0}=\frac{N-\mu+2}{2(2 N-\mu)} S_{H, L}^{\frac{2 N-\mu}{N-\mu+2}}$.

Notations: Throughout the paper we will use the notation $\mathcal{J}_{0}=\mathcal{J}, \mathcal{N}_{0}=\mathcal{N},\|\cdot\|=\|\cdot\|_{H_{0}^{1}(\Omega)}$

$$
a(u)=\int_{\Omega}|\nabla u|^{2} d x \text { and } b(u)=\iint_{\Omega} \frac{\left(u^{+}(x)\right)^{2_{\mu}^{*}}\left(u^{+}(y)\right)^{2_{\mu}^{*}}}{|x-y|^{\mu}} d x d y .
$$

An easy consequence of (2.1) gives $\partial_{f}$ is coercive and bounded below on $\mathcal{N}_{f}$.

Proposition 2.6. For any $u, v \in H_{0}^{1}(\Omega)$, we have

$$
\int_{\Omega} \int_{\Omega} \frac{|u(x)|^{2_{\mu}^{*}}|v(y)|^{2_{\mu}^{*}}}{\left.|x-y|\right|^{\mu}} d x d y \leq\left(\int_{\Omega} \frac{|u(x)|_{\Omega}^{2_{\mu}^{*}}|u(y)|^{2_{\mu}^{*}}}{\left.|x-y|\right|^{\mu}} d x d y\right)^{\frac{1}{2}}\left(\int_{\Omega} \int_{\Omega} \frac{|v(x)|^{2_{\mu}^{*}}|v(y)|^{2_{\mu}^{*}}}{|x-y|^{\mu}} d x d y\right)^{\frac{1}{2}} .
$$

Proof. For details of the proof, see [17, Lemma 2.3].

Lemma 2.7. For each $u \in H_{0}^{1}(\Omega)$, there exists a unique $t>0$ such that $t u \in \mathcal{N}$. Moreover, there holds $\Upsilon_{0} \leq\left(\frac{N-\mu+2}{2(2 N-\mu)}\right)\left(\frac{a(u)^{2 *}}{b(u)}\right)^{\frac{1}{2_{\mu}^{*}-1}}$.

Proof. Let $m_{u}(t)=\frac{t^{2}}{2} a(u)-\frac{t^{2 \cdot 2_{\mu}^{*}}}{2 \cdot 2_{\mu}^{*}} b(u)$ then on solving $m_{u}^{\prime}(t)=0$, we get unique $t(u)=\left(\frac{a(u)}{b(u)}\right)^{\frac{1}{2\left(2_{\mu}^{*}-1\right)}}$ such that $t(u) u \in \mathcal{N}$. From the definition of $\Upsilon_{0}$, we have

$$
\Upsilon_{0} \leq \mathcal{J}(t(u) u)=\left(\frac{1}{2}-\frac{1}{2 \cdot 2_{\mu}^{\star}}\right)\left(\frac{a(u)}{b(u)}\right)^{\frac{1}{2 \mu^{*}-1}} a(u)=\left(\frac{N-\mu+2}{2(2 N-\mu)}\right)\left(\frac{a(u)^{2_{\mu}^{\star}}}{b(u)}\right)^{\frac{1}{2_{\mu}^{*}-1}} .
$$

Remark 2.8. We remark that by [15, Lemma 1.3], $S_{H, L}$ is never achieved on bounded domain. Therefore if $u$ is a solution of the following equation

$$
-\Delta u=\left(\int_{\Omega} \frac{|u(y)|^{2_{\mu}^{*}}}{|x-y|^{\mu}} d y\right)|u|^{2_{\mu}^{*}-2} u \text { in } \Omega, \quad u=0 \text { on } \partial \Omega,
$$

then $\mathcal{J}(u)>\Upsilon_{0}=\frac{N-\mu+2}{2(2 N-\mu)} S_{H, L}^{\frac{2 N-\mu}{N-\mu+2}}$.

Lemma 2.9. A sequence $\left\{u_{n}\right\}$ is a $(P S)_{\Upsilon_{0}}$ - sequence for $\mathcal{J}$ in $H_{0}^{1}(\Omega)$ if and only if $\mathcal{J}\left(u_{n}\right)=\Upsilon_{0}+o_{n}(1)$ and $a\left(u_{n}\right)=b\left(u_{n}\right)+o_{n}(1)$.

Proof. Clearly, any $(P S)_{\Upsilon_{0}}$ - sequence satisfies $a\left(u_{n}\right)=b\left(u_{n}\right)+o_{n}(1)$ and $\mathcal{J}\left(u_{n}\right)=\Upsilon_{0}+o_{n}(1)$. Conversely, let $\mathcal{J}\left(u_{n}\right)=\Upsilon_{0}+o_{n}(1)$ and $a\left(u_{n}\right)=b\left(u_{n}\right)+o_{n}(1)$ then $\Upsilon_{0}=\mathcal{J}\left(u_{n}\right)=\frac{N-\mu+2}{2(2 N-\mu)} b\left(u_{n}\right)+o_{n}(1)$ and hence we have

$$
b\left(u_{n}\right)=D \Upsilon_{0}+o_{n}(1) \text { where } D=\frac{2(2 N-\mu)}{N-\mu+2} .
$$


Define $T_{n}(\psi)=\iint_{\Omega} \frac{\left(u_{n}^{+}(x)\right)^{2_{\mu}^{*}}\left(u_{n}^{+}(y)\right)^{2_{\mu}^{*}-1} \psi(y)}{|x-y|^{\mu}} d x d y$ for $\psi \quad \in \quad H_{0}^{1}(\Omega)$ and $n=1,2, \cdots$.

Claim: $\left\|T_{n}\right\|_{H^{-1}}=\left(D \Upsilon_{0}\right)^{\frac{1}{2}}+o_{n}(1)$.

Let $\psi \in H_{0}^{1}(\Omega)$ such that $\|\psi\|=1$ then by Lemma 2.7, we know that there exists a $t>0$ such that $a(t \psi)=b(t \psi)$. Therefore, $t=\|\psi\|_{N L}^{-\frac{2_{\mu}^{*}}{2_{\mu-1}^{*}}}$ and $\Upsilon_{0} \leq \frac{1}{D}\|\psi\|_{N L}^{-\frac{2.2_{\mu}^{*}}{2_{\mu}^{*}-1}}$. This implies,

$$
\|\psi\|_{N L} \leq\left(\frac{1}{D \Upsilon_{0}}\right)^{\frac{2_{\mu}^{*}-1}{2 \cdot 2_{\mu}^{*}}} .
$$

Taking into account (2.4), (2.5), Proposition 2.6 and employing Hölder's inequality, for each $n$, we have

$$
\begin{aligned}
\left|T_{n}(\psi)\right| & \leq\left(\int_{\Omega} \int_{\Omega} \frac{\left(u_{n}^{+}(x)\right)^{2_{\mu}^{*}}\left(u_{n}^{+}(y)\right)^{2_{\mu}^{*}}}{|x-y|^{\mu}} d x d y\right)^{\frac{2 \cdot 2_{\mu}^{*}-1}{2 \cdot 2_{\mu}^{*}}}\left(\int_{\Omega} \int_{\Omega} \frac{|\psi(x)|^{2_{\mu}^{*}}|\psi(y)|^{2_{\mu}^{*}}}{|x-y|^{\mu}} d x d y\right)^{\frac{1}{2 \cdot 2_{\mu}^{*}}} \\
& =b\left(u_{n}\right)^{\frac{2 \cdot 2^{*}{ }^{*}-1}{2 \cdot 2_{\mu}^{*}}}\|\psi\|_{N L} \\
& \leq\left(\frac{1}{D \Upsilon_{0}}\right)^{\frac{2_{\mu}^{*}-1}{2 \cdot 2_{\mu}^{*}}}\left(D \Upsilon_{0}+o_{n}(1)\right)^{\frac{2 \cdot 2_{\mu}^{*}-1}{2 \cdot 2_{\mu}^{*}}}=\left(D \Upsilon_{0}\right)^{\frac{1}{2}}+o_{n}(1) \text { as } n \rightarrow \infty .
\end{aligned}
$$

So, we get $\left\|T_{n}\right\|_{H^{-1}} \leq\left(D \Upsilon_{0}\right)^{\frac{1}{2}}+o_{n}(1)$. Moreover, $T_{n}\left(\frac{u_{n}}{\left\|u_{n}\right\|}\right)=\left(b\left(u_{n}\right)\right)^{\frac{1}{2}}=\left(D \Upsilon_{0}\right)^{\frac{1}{2}}+o_{n}(1)$. This implies $\left\|T_{n}\right\|_{H^{-1}}=$ $\left(D \Upsilon_{0}\right)^{\frac{1}{2}}+o_{n}(1)$. Hence the proof of claim follows. Now, by Riesz representation theorem, for each $n$, there exists $v_{n} \in H_{0}^{1}(\Omega)$ such that

$$
T_{n}(\psi)=\left\langle v_{n}, \psi\right\rangle=\int_{\Omega} \nabla v_{n} \cdot \nabla \psi d x \text { and }\left\|v_{n}\right\|=\left\|T_{n}\right\|_{H^{-1}}=\left(D \Upsilon_{0}\right)^{\frac{1}{2}}+o_{n}(1) .
$$

Thus, $\left\langle v_{n}, u_{n}\right\rangle=T_{n}\left(u_{n}\right)=b\left(u_{n}\right)=D \Upsilon_{0}+o_{n}(1)$. Hence,

$$
\begin{aligned}
\left\|u_{n}-v_{n}\right\|^{2} & =\left\|u_{n}\right\|^{2}-2\left\langle u_{n}, v_{n}\right\rangle+\left\|v_{n}\right\|^{2} \\
& =D \Upsilon_{0}-2 D \Upsilon_{0}+D \Upsilon_{0}+o_{n}(1)=o_{n}(1) \text { as } n \rightarrow \infty .
\end{aligned}
$$

For any $\psi \in H_{0}^{1}(\Omega)$ with $\|\psi\|=1$, we have

$$
\left\langle g^{\prime}\left(u_{n}\right), \psi\right\rangle=\int_{\Omega} \nabla u_{n} \cdot \nabla \psi d x-T_{n}(\psi)=\left\langle u_{n}, \psi\right\rangle-\left\langle v_{n}, \psi\right\rangle=\left\langle u_{n}-v_{n}, \psi\right\rangle .
$$

Therefore, $\left\|\mathcal{J}^{\prime}\left(u_{n}\right)\right\|_{H^{-1}} \leq\left\|u_{n}-v_{n}\right\|=o_{n}(1)$. It implies $J^{\prime}\left(u_{n}\right) \rightarrow 0$ in $H^{-1}$.

Clearly, $\mathcal{N}_{f}$ contains every non zero solution of $\left(P_{f}\right)$ and we know that the Nehari manifold is closely related to the behavior of the fibering maps $\phi_{u}: \mathbb{R}^{+} \rightarrow \mathbb{R}$ defined as $\phi_{u}(t)=\mathcal{J}_{f}(t u)$. It is easy to see that $t u \in \mathcal{N}_{f}$ if and only if $\phi_{u}^{\prime}(t)=0$ and elements of $\mathcal{N}_{f}$ correspond to stationary points of the fibering maps. It is natural to divide $\mathcal{N}_{f}$ into the following sets

$$
\mathcal{N}_{f}^{+}=:\left\{u \in \mathcal{N}_{f} \mid \phi_{u}^{\prime \prime}(1)>0\right\}, \mathcal{N}_{f}^{-}=:\left\{u \in \mathcal{N}_{f} \mid \phi_{u}^{\prime \prime}(1)<0\right\} \text {, and } \mathcal{N}_{f}^{0}=:\left\{u \in \mathcal{N}_{f} \mid \phi_{u}^{\prime \prime}(1)=0\right\} .
$$

We also denote the infimum over $\mathcal{N}_{f}^{+}$and $\mathcal{N}_{f}^{-}$as

$$
\Upsilon_{f}^{+}=\inf _{u \in \mathcal{N}_{f}^{+}} \partial_{f}(u) \quad \Upsilon_{f}^{-}=\inf _{u \in \mathcal{N}_{f}^{-}} \mathcal{J}_{f}(u) .
$$




\section{Existence of First Solution}

In this section we prove the existence of first solution by showing the existence of minimizer for $\mathcal{J}_{f}$ over the Nehari manifold $\mathcal{N}_{f}$. First we state some Lemmas whose proof can be found in [30]. We further prove some properties of the manifold $\mathcal{N}_{f}^{+}$.

Lemma 3.1. If $f \in \hat{F}$ and $\|f\|_{H^{-1}}<e_{00}:=C_{N, \mu} S_{H, L}^{\frac{2_{\mu}^{*}}{2.2_{\mu}^{*}-2}}$ where $C_{N, \mu}=\left(\frac{1}{2.2_{\mu}^{*}-1}\right)^{\frac{2.2_{\mu}^{*}-1}{2.2_{\mu}^{*}-2}}\left(2.2_{\mu}^{\star}-2\right)$ then $\alpha_{0}:=\inf _{u \in E}\left\{C_{N, \mu}\|u\|^{\frac{2.2_{\mu}^{*}-1}{2_{\mu}^{*}-1}}-\int_{\Omega} f u d x\right\}$ is acheived, where

$$
E:=\left\{u \in H_{0}^{1}(\Omega): \int_{\Omega} \int_{\Omega} \frac{|u(x)|^{2_{\mu}^{*}}|u(y)|^{2_{\mu}^{*}}}{|x-y|^{\mu}} d x d y=1\right\} .
$$

Proof. Proof follows from [30, Lemma 4.1]. Since we consider $\lambda=0$ in equation (4.1) of [30], our result holds for all $N \geq 3$.

Lemma 3.2. For every $u \in \mathcal{N}_{f}, u \neq \equiv$ we have $a(u)-\left(2.2_{\mu}^{\star}-1\right) b(u) \neq 0$. In particular, $\mathcal{N}_{f}^{0}=\{0\}$.

Lemma 3.3. For each $u \in H_{0}^{1}(\Omega)$ with $u^{+} \not \equiv 0$ the following holds:

(a) There exists a unique $t^{-}=t^{-}(u)>0$ such that $t^{-} u \in \mathcal{N}_{f}^{-}(\Omega)$. In particular,

$$
t^{-}>\left(\frac{a(u)}{\left(2.2_{\mu}^{\star}-1\right) b(u)}\right)^{\frac{1}{2.2_{\mu}^{*}-2}}:=t_{\max }
$$

and $\mathcal{J}_{f}\left(t^{-} u\right)=\max _{t \geq t_{\max }} \mathcal{J}_{f}(t u)$.

(b) If $\int_{\Omega} f u>0$, then there exists unique $t^{+} \in\left(0, t_{\max }\right)$ such that $t^{+} u \in \mathcal{N}_{f}^{+}(\Omega)$ and

$$
\partial_{f}\left(t^{+} u\right)=\min _{0<t \leq t^{-}} \mathcal{J}_{f}(t u)
$$

(c) $t^{-}(u)$ is a continuous function.

(d) $\mathcal{N}_{f}^{-}=\left\{u \in H_{0}^{1}(\Omega) \backslash\{0\} \mid u^{+} \not \equiv 0\right.$ and $\left.\frac{1}{\|u\|} t^{-}\left(\frac{u}{\|u\|}\right)=1\right\}$.

Lemma 3.4. For each $u \in \mathcal{N}_{f}^{+}(\Omega)$, we have $\int_{\Omega} f u d x>0$ and $\mathcal{J}_{f}(u)<0$. In particular, $\Upsilon_{f}(\Omega) \leq \Upsilon_{f}^{+}(\Omega)<0$.

Lemma 3.5. Let $u \in \mathcal{N}_{f}(\Omega)$ be such that $\mathcal{J}_{f}(u)=\min _{w \in \mathcal{N}_{f}(\Omega)} \mathcal{J}_{f}(w)=\Upsilon_{f}(\Omega)$ then $\int_{\Omega} f u d x>0$ and $u$ is a solution of $\left(P_{f}\right)$.

Lemma 3.6. $J_{f}$ has Palais-Smale sequences at each of the levels $\Upsilon_{f}(\Omega), \Upsilon_{f}^{+}(\Omega)$ and $\Upsilon_{f}^{-}(\Omega)$.

Lemma 3.7. Let $\left\{u_{n}\right\} \in \mathcal{N}_{f}$ be a $(P S)_{\Upsilon_{f}(\Omega)}$ sequence for $\mathcal{J}_{f}$, then there exists a subsequence of $\left\{u_{n}\right\}$, still denoted by $\left\{u_{n}\right\}$, and a non-zero $u_{1} \in H_{0}^{1}(\Omega)$ such that $u_{n} \rightarrow u_{1}$ strongly in $H_{0}^{1}(\Omega)$. Moreover, $u_{1} \in \mathcal{N}_{f}$ and is a solution to $\left(P_{f}\right)$.

Proof. $\partial_{f}$ is bounded below and coercive implies $\left\{u_{n}\right\}$ is bounded in $H_{0}^{1}(\Omega)$. So, there exists a subsequence still denoted by $\left\{u_{n}\right\}$ such that $u_{n} \rightarrow u_{1}$ weakly in $H_{0}^{1}(\Omega)$. By [19, Lemma 4.2], we have $\mathcal{J}_{f}^{\prime}\left(u_{1}\right)=0$. In particular, $u_{1} \in \mathcal{N}_{f}$ and $\mathcal{J}_{f}\left(u_{1}\right)=\left(\frac{1}{2}-\frac{1}{2.2^{\star}{ }_{\mu}}\right) a\left(u_{1}\right)-\left(1-\frac{1}{2.2^{\star} \mu}\right) \int_{\Omega} f u_{1} d x$. Now, using the fact that $a$ is weakly lower semi continuous we have

$$
\Upsilon_{f}(\Omega) \leq \partial_{f}\left(u_{1}\right) \leq \liminf _{n \rightarrow \infty}\left(\frac{1}{2}-\frac{1}{2.2^{\star} \mu}\right) a\left(u_{n}\right)-\lim _{n \rightarrow \infty}\left(1-\frac{1}{2 \cdot 2^{\star} \mu}\right) \int_{\Omega} f u_{n} d x=\Upsilon_{f}(\Omega) .
$$


Consequently, we have $\Upsilon_{f}(\Omega)=\mathcal{J}_{f}\left(u_{1}\right)$. Let $w_{n}=u_{n}-u_{1}$ then by [19, Lemma 4.1], [15, Lemma 2.2] and the fact that $\mathcal{J}_{f}^{\prime}\left(u_{1}\right)=0$, we obtain $\mathcal{J}_{f}\left(w_{n}\right)=\mathcal{J}_{f}\left(u_{n}\right)-\mathcal{J}_{f}\left(u_{1}\right)=o_{n}(1)$ and $\left\langle\mathcal{J}_{f}^{\prime}\left(w_{n}\right), \phi\right\rangle=\left\langle\mathcal{J}_{f}^{\prime}\left(u_{n}\right), \phi\right\rangle-\left\langle\mathcal{J}_{f}^{\prime}\left(u_{1}\right), \phi\right\rangle+o_{n}(1)=$ $o_{n}(1)$. Therefore, $\left\langle\partial_{f}^{\prime}\left(w_{n}\right), w_{n}\right\rangle=o_{n}(1)$. It implies $\partial_{f}\left(w_{n}\right)=\left(\frac{1}{2}-\frac{1}{2.2^{\star} \mu}\right) a\left(w_{n}\right)-\int_{\Omega} f w_{n} d x=o_{n}(1)$ and since $\int_{\Omega} f w_{n} d x=o_{n}(1)$, we get $a\left(w_{n}\right)=o_{n}(1)$. Hence $u_{n} \rightarrow u$ strongly in $H_{0}^{1}(\Omega)$.

Lemma 3.8. If $u$ be a solution of $\left(P_{f}\right)$ then $u \in C^{2}(\bar{\Omega})$. Moreover, $u$ is a positive solution.

Proof. Let $u$ be a solution of $\left(P_{f}\right)$ and $G(x, u)=\left(\int_{\Omega} \frac{\left|u^{+}(y)\right|^{2_{\mu}^{*}}}{|x-y|^{\mu}} d y\right)\left|u^{+}\right|^{2_{\mu^{*}}^{-}} u+f$. By using same assertions and arguments as in [25, Proposition 3.1 and Theorem 2], we have $\left(\int_{\Omega} \frac{\left|u^{+}(y)\right|^{2_{\mu}^{*}}}{|x-y|^{\mu}} d y\right) \in L^{\infty}(\Omega)$ and since $f \in \hat{F}$, we have $|G(x, u)| \leq C\left(1+|u|^{2^{*}-1}\right)$. Then by the standard elliptic regularity $u \in C^{2}(\bar{\Omega})$. Since $f \geq 0$, we get $u \geq 0$ and by using strong maximum principle, $u$ is a positive solution of $\left(P_{f}\right)$.

Lemma 3.9. Let $\mu<\min \{4, N\}$ and $k_{0}=\left(\frac{1}{2.2_{\mu}^{*}-1}\right)^{\frac{1}{2\left(2_{\mu}^{*}-1\right)}} S_{H, L}^{\frac{2_{\mu}^{*}}{2\left(2 \mu_{\mu}^{*}-1\right)}}$ and $f \in \hat{F},\|f\|_{H^{-1}} \leq e_{00}$ (where $e_{00}$ is defined in Lemma 3.1) then

1. $\mathcal{N}_{f}^{+}(\Omega) \subset B_{k_{0}}(0):=\left\{u \in H_{0}^{1}(\Omega) \mid\|u\|<k_{0}\right\}$.

2. $\partial_{f}$ is strictly convex in $B_{k_{0}}(0)$.

\section{Proof.}

1. Let $u \in \mathcal{N}_{f}^{+}(\Omega)$ then $\phi_{u}^{\prime}(1)=0$ and $\phi_{u}^{\prime \prime}(1)>0$. That is, $a(u)=b(u)+\int_{\Omega} f u d x$ and $a(u)>\left(2.2_{\mu}^{\star}-1\right) b(u)$. Therefore, $a(u)=b(u)+\int_{\Omega} f u d x<\frac{1}{\left(2.2_{\mu}^{*}-1\right)} a(u)+\int_{\Omega} f u d x$. It implies $\left(1-\frac{1}{\left(2.2_{\mu}^{*}-1\right)}\right) a(u) \leq\|f\|_{H^{-1}}\|u\|$. So,

$$
\begin{aligned}
\|u\| & \leq \frac{\left(2.2_{\mu}^{\star}-1\right)}{2\left(2_{\mu}^{\star}-1\right)}\|f\|_{H^{-1}} \\
& \leq \frac{\left(2.2_{\mu}^{\star}-1\right)}{2\left(2_{\mu}^{\star}-1\right)} C_{N, \mu} S_{H, L}^{\frac{2_{\mu}^{*}}{2.2_{\mu}^{-2}}}=\left(\frac{1}{2 \cdot 2_{\mu}^{\star}-1}\right)^{\frac{1}{2\left(2_{\mu}^{*}-1\right)}} S_{H, L}^{\frac{2_{\mu}^{*}}{2\left(2_{\mu}^{*}-1\right)}}=k_{0} .
\end{aligned}
$$

2. By using Hölders inequality and equation (2.2), we have

$$
\begin{aligned}
\iint_{\Omega} \frac{\left(u^{+}(x)\right)^{2_{\mu}^{*}-1}\left(u^{+}(y)\right)^{2_{\mu}^{*}-1} z(x) z(y)}{|x-y|^{\mu}} d x d y & \leq b(u)^{\frac{2_{\mu}^{*}-1}{2_{\mu}^{*}}}\|z\|_{N L}^{2} \\
& \leq S_{H, L}^{-\left(2_{\mu}^{*}-1\right)} a(u)^{\left(2_{\mu}^{*}-1\right)} S_{H, L}^{-1} a(z) \\
& =S_{H, L}^{-2_{\mu}^{*}} a(u)^{\left(2_{\mu}^{*}-1\right)} a(z) .
\end{aligned}
$$

Again using Hölders inequality, Proposition 2.6 and (2.2), we have

$$
\iint_{\Omega} \frac{\left(u^{+}(x)\right)^{2_{\mu}^{*}}\left(u^{+}(y)\right)^{2_{\mu}^{*}-2} z^{2}(y)}{|x-y|^{\mu}} d x d y \leq b(u)^{\frac{2_{\mu}^{*}-1}{2_{\mu}^{*}}}\|z\|_{N L}^{2} \leq S_{H, L}^{-2_{\mu}^{*}} a(u)^{\left(2_{\mu}^{*}-1\right)} a(z) .
$$


From equations (3.1), (3.2) and definition of $\mathcal{\partial}_{f}^{\prime \prime}(u)(z, z)$, we get

$$
\begin{aligned}
J_{f}^{\prime \prime}(u)(z, z)= & a(z)-2_{\mu}^{\star} \int_{\Omega} \int_{\Omega} \frac{\left(u^{+}(x)\right)^{2_{\mu}^{*}-1}\left(u^{+}(y)\right)^{2_{\mu}^{*}-1} z(x) z(y)}{|x-y|^{\mu}} d x d y \\
& -\left(2_{\mu}^{\star}-1\right) \iint_{\Omega} \frac{\left(u^{+}(x)\right)^{2_{\mu}^{*}}\left(u^{+}(y)\right)^{2_{\mu}^{*}-2} z^{2}(y)}{|x-y|^{\mu}} d x d y \\
\geq & a(z)\left(1-2_{\mu}^{\star} S_{H, L}^{-2_{\mu}^{*}} a(u)^{\left(2_{\mu}^{*}-1\right)}-\left(2_{\mu}^{\star}-1\right) S_{H, L}^{-2_{\mu}^{*}} a(u)^{\left(2_{\mu}^{*}-1\right)}\right) \\
= & a(z)\left(1-\left(2.2_{\mu}^{\star}-1\right) S_{H, L}^{-2_{\mu}^{*}} a(u)^{\left(2_{\mu}^{*}-1\right)}\right) \\
> & a(z)\left(1-\frac{\left(2.2_{\mu}^{\star}-1\right)}{\left(2.2_{\mu}^{\star}-1\right)}\right)=0
\end{aligned}
$$

for $u \in B_{k_{0}}(0) \backslash\{0\}$. Then $\mathcal{J}_{f}^{\prime \prime}(u)$ is positive definite for $u \in B_{k_{0}}(0)$ and $\mathcal{J}_{f}(u)$ is strictly convex on $B_{k_{0}}(0)$.

Lemma 3.10. It holds that $u_{1} \in \mathcal{N}_{f}^{+}$and $\mathcal{J}_{f}\left(u_{1}\right)=\Upsilon_{f}^{+}(\Omega)=\Upsilon_{f}(\Omega)$. Moreover, $u_{1}$ is the unique critical point of $\mathcal{J}_{f}$ in $B_{k_{0}}(0)$ and $u_{1}$ is a local minimum of $\mathcal{J}_{f}$ in $H_{0}^{1}(\Omega)$.

Proof. Using the proof of [30, Theorem 1.3], we have $\int_{\Omega} f u_{1} d x>0$. Now if $u_{1} \in \mathcal{N}_{f}^{-}$then there exists a unique $t^{-}\left(u_{1}\right)=1>t_{\max }>t^{+}\left(u_{1}\right)>0$ such that $t^{+}\left(u_{1}\right) u_{1} \in \mathcal{N}_{f}^{+}$then by Lemma $3.3(\mathrm{~b})$ we have

$$
\Upsilon_{f}(\Omega) \leq \Upsilon_{f}^{+}(\Omega) \leq \partial_{f}\left(t^{+}\left(u_{1}\right) u_{1}\right)<\partial_{f}\left(t^{-}\left(u_{1}\right) u_{1}\right)=\partial_{f}\left(u_{1}\right)=\Upsilon_{f}(\Omega) .
$$

which is a contradiction. It implies $u_{1} \in \mathcal{N}_{f}^{+}$and $\Upsilon_{f}^{+}(\Omega) \leq \mathcal{J}_{f}\left(u_{1}\right)=\Upsilon_{f}(\Omega) \leq \Upsilon_{f}^{+}(\Omega)$ that is, $\mathcal{J}_{f}\left(u_{1}\right)=\Upsilon_{f}(\Omega)=$ $\Upsilon_{f}^{+}(\Omega)$. Using Lemma 3.5 and Lemma 3.9, we get $u_{1}$ is the unique critical point of $\mathcal{J}_{f}$ in $B_{k_{0}}(0)$ and the proof of local minimum follows from [30, Lemma 3.2].

Lemma 3.11. Let $\mu<\min \{4, N\}$ and $u \in H_{0}^{1}(\Omega)$ be a critical point of $\mathcal{J}_{f}$ then either $u \in \mathcal{N}_{f}^{-}$or $u=u_{1}$.

Proof. If $u \in H_{0}^{1}(\Omega)$ be a critical point of $\mathcal{\partial}_{f}$ then $u \in \mathcal{N}_{f}=\mathcal{N}_{f}^{+} \cup \mathcal{N}_{f}^{-}$. Now using the fact that $\mathcal{N}_{f}^{+} \cap \mathcal{N}_{f}^{-}=\emptyset$ and $\mathcal{N}_{f}^{+} \subset B_{k_{0}}(0)$ we have either $u \in \mathcal{N}_{f}^{-}$or $u=u_{1}$.

\section{Asymptotic estimates and Palais-Smale Analysis}

In this section we shall prove that the functional $\partial_{f}$ satisfies Palais-Smale condition strictly below the first critical level and (strictly) between the first and second critical levels. To start with, we shall prove several new estimates on the nonlinearity.

It is known from Lemma 2.2 that the best constant $S_{H, L}$ is achieved by the function

$$
u(x)=S^{\frac{(N-\mu)(2-N)}{4(N-\mu+2)}}(C(N, \mu))^{\frac{2-N}{2(N-\mu+2)}} \frac{(N(N-2))^{\frac{N-2}{4}}}{\left(1+|x|^{2}\right)^{\frac{N-2}{2}}},
$$

which is a solution of the problem $-\Delta u=\left(|x|^{-\mu \star}|u|^{2_{\mu}^{\star}}\right)|u|^{2_{\mu}^{*}-1}$ in $\mathbb{R}^{N}$ with

$$
\int_{\mathbb{R}^{N}}|\nabla u|^{2} d x=\iint_{\mathbb{R}^{N}} \frac{|u(x)|_{\mathbb{R}^{N}}^{2^{*}}|u(y)|^{2^{*}}}{|x-y|^{\mu}} d x d y=S_{H, L}^{\frac{2 N-\mu}{N-\mu+2}} .
$$


We may assume $R_{1}=\rho, R_{2}=1 / \rho$ for $\rho \in\left(0, \frac{1}{2}\right)$. Now, define $v_{\rho} \in C_{c}^{\infty}\left(\mathbb{R}^{N}\right)$ such that $0 \leq v_{\rho}(x) \leq 1$ for all $x \in \mathbb{R}^{N}$, radially symmetric and

$$
v_{\rho}(x)= \begin{cases}0 & 0<|x|<\frac{3 \rho}{2}, \\ 1 & 2 \rho \leq|x| \leq \frac{1}{2 \rho}, \\ 0 & |x| \geq \frac{3}{4 \rho},\end{cases}
$$

and

$$
u_{\sigma}^{\epsilon}(x)=S^{\frac{(N-\mu)(2-N)}{4(N-\mu+2)}} C(N, \mu)^{\frac{2-N}{2(N-\mu+2)}} \frac{\left(N(N-2) \epsilon^{2}\right)^{\frac{N-2}{4}}}{\left(\epsilon^{2}+|x-(1-\epsilon) \sigma|^{2}\right)^{\frac{N-2}{2}}},
$$

where $\sigma \in \mathbb{S}^{N-1}:=\left\{x \in \mathbb{R}^{N}:|x|=1\right\}, 0<\epsilon \leq 1$. Set

$$
g_{\rho}^{\epsilon, \sigma}(x):=v_{\rho}(x) u_{\sigma}^{\epsilon}(x) \in H_{0}^{1}(\Omega) .
$$

Lemma 4.1. (i) $a\left(g_{\rho}^{\epsilon, \sigma}\right)=b\left(g_{\rho}^{\epsilon, \sigma}\right)=S_{H, L}^{\frac{2 N-\mu}{N-\mu+2}}+o_{\epsilon}(1)$ uniformly in $\sigma$ as $\epsilon \rightarrow 0$.

(ii) $\mathcal{J}\left(g_{\rho}^{\epsilon, \sigma}\right)=\frac{N-\mu+2}{2(2 N-\mu)} S_{H, L}^{\frac{2 N-\mu}{N-\mu+2}}+o_{\epsilon}(1)$ uniformly in $\sigma$ as $\epsilon \rightarrow 0$.

(iii) $g_{\rho}^{\epsilon, \sigma} \rightarrow 0$ weakly in $H_{0}^{1}(\Omega)$ uniformly in $\sigma$ as $\epsilon \rightarrow 0$.

Proof.

(i) Observe the fact that there exist constants $d_{1}, d_{2}>0$ such that

$$
\begin{aligned}
d_{1}<|x-(1-\epsilon) \sigma|< & d_{2} \text { for all } x \in B_{2 \rho} \text { whenever } \epsilon<1-2 \rho . \\
\left\|\nabla g_{\rho}^{\epsilon, \sigma}\right\|_{L^{2}\left(\mathbb{R}^{N}\right)}-\left\|\nabla u_{\epsilon}^{\sigma}\right\|_{L^{2}\left(\mathbb{R}^{N}\right) \leq} & \int_{\left(\mathbb{R}^{N} \backslash B_{\frac{1}{2 \rho}}\right) \cup B_{2 \rho}}\left|\nabla u_{\epsilon}^{\sigma}\right|^{2} d x+\rho^{-2} \int_{B_{2 \rho}}\left|u_{\epsilon}^{\sigma}\right|^{2} d x \\
& +\rho^{2} \int_{B_{\frac{3}{4 \rho}} \backslash B_{\frac{1}{2 \rho}}}\left|u_{\epsilon}^{\sigma}\right|^{2} d x \\
\leq & C \epsilon^{N-2} \int_{\left(\mathbb{R}^{N} \backslash B_{\frac{1}{2 \rho}}\right) \cup B_{2 \rho}} \frac{|x-(1-\epsilon) \sigma|^{2}}{|x-(1-\epsilon) \sigma|^{2 N}} d x \\
& +C \epsilon^{N-2} \int_{B_{2 \rho} \cup B_{\frac{3}{4 \rho}} \backslash B_{\frac{1}{2 \rho}}} \frac{d x}{|x-(1-\epsilon) \sigma|^{2(N-2)}}=O\left(\epsilon^{N-2}\right) .
\end{aligned}
$$


Thus, $\left\|\nabla g_{\rho}^{\epsilon, \sigma}\right\|_{L^{2}\left(\mathbb{R}^{N}\right)}=\left\|\nabla u_{\epsilon}^{\sigma}\right\|_{L^{2}\left(\mathbb{R}^{N}\right)}+o_{\epsilon}(1)=S_{H, L}^{\frac{2 N-\mu}{N-\mu+2}}+o_{\epsilon}(1)$.

Next we will prove that $b\left(g_{\rho}^{\epsilon, \sigma}\right)=S_{H, L}^{\frac{2 N-\mu}{N-\mu+2}}+o_{\epsilon}(1)$ uniformly in $\sigma$ as $\epsilon \rightarrow 0$. For this consider

$$
\begin{aligned}
\int & \int_{\mathbb{R}^{N}} \frac{\left|g_{\mathbb{R}^{N}}^{\epsilon, \sigma}(x)\right|^{2_{\mu}^{*}}\left|g_{\rho}^{\epsilon, \sigma}(y)\right|^{2_{\mu}^{*}}}{|x-y|^{\mu}} d x d y-\int_{\mathbb{R}^{N}} \int_{\mathbb{R}^{N}} \frac{\left|u_{\epsilon}^{\sigma}(x)\right|^{2_{\mu}^{*}}\left|u_{\epsilon}^{\sigma}(y)\right|^{2_{\mu}^{*}}}{|x-y|^{\mu}} d x d y \\
= & \int_{\mathbb{R}^{N}} \int_{\mathbb{R}^{N}} \frac{\left(\left|v_{\rho}(x)\right|^{2_{\mu}^{*}}\left|v_{\rho}(y)\right|^{2_{\mu}^{*}}-1\right)\left|u_{\epsilon}^{\sigma}(x)\right|^{2_{\mu}^{*}}\left|u_{\epsilon}^{\sigma}(y)\right|^{2_{\mu}^{*}}}{\left.|x-y|\right|^{\mu}} d x d y \\
\leq & C\left(\int_{B_{2 \rho}} \int_{B_{2 \rho}}+\int_{B_{\frac{1}{2 \rho}} \backslash B_{2 \rho}} \int_{B_{2 \rho}}+\int_{B_{\frac{1}{2 \rho}} \backslash B_{2 \rho}} \int_{\mathbb{R}^{N} \backslash B_{\frac{1}{2 \rho}}}\right. \\
& \left.+\int_{\mathbb{R}^{N} \backslash B_{\frac{1}{2 \rho}}} \int_{B_{2 \rho}}+\int_{\mathbb{R}^{N} \backslash B_{\frac{1}{2 \rho}}} \int_{\mathbb{R}^{N} \backslash B_{\frac{1}{2 \rho}}}\right)_{\frac{\left|u_{\epsilon}^{\sigma}(x)\right|^{2_{\mu}^{*}}\left|u_{\epsilon}^{\sigma}(y)\right|^{2_{\mu}^{*}}}{|x-y|^{\mu}}} d x d y, \\
= & C \sum_{i=1}^{i=5} J_{i},
\end{aligned}
$$

Let $\xi_{\epsilon}(x)=\frac{\epsilon^{N}}{\left(\epsilon^{2}+|x-(1-\epsilon) \sigma|^{2}\right)^{N}}$ then taking into account the definition of $u_{\epsilon}^{\sigma}$, (4.2) and Hardy-LittlewoodSobolev inequality, we have the following estimates:

$$
\begin{aligned}
& J_{1} \leq C(N, \mu)\left(\int_{B_{2 \rho}} S^{\frac{-N(N-\mu)}{2(N-\mu+2)}} C(N, \mu)^{\frac{-N}{(N-\mu+2)}}(N(N-2))^{\frac{N}{2}} \xi_{\epsilon}(x) d x\right)^{\frac{2 N-\mu}{N}} \\
& \leq C \epsilon^{2 N-\mu}\left(\int_{B_{2 \rho}} \frac{d x}{|x-(1-\epsilon) \sigma|^{2 N}}\right)^{\frac{2 N-\mu}{N}} \leq C \epsilon^{2 N-\mu}\left(\int_{B_{2 \rho}} d x\right)^{\frac{2 N-\mu}{N}}=O\left(\epsilon^{2 N-\mu}\right), \\
& J_{2} \leq C\left(\int_{B_{\frac{1}{2 \rho}} \backslash B_{2 \rho}} \xi_{\epsilon}(x) d x\right)^{\frac{2 N-\mu}{2 N}}\left(\int_{B_{2 \rho}} \xi_{\epsilon}(x) d x\right)^{\frac{2 N-\mu}{2 N}} \\
& \leq C \epsilon^{\frac{2 N-\mu}{2}}\left(\int_{B_{2 \rho}} \frac{d x}{|x-(1-\epsilon) \sigma|^{2 N}}\right)^{\frac{2 N-\mu}{2 N}}=O\left(\epsilon^{\frac{2 N-\mu}{2}}\right), \\
& J_{3} \leq C\left(\int_{B_{\frac{1}{2 \rho}} \backslash B_{2 \rho}} \xi_{\epsilon}(x)\right)^{\frac{2 N-\mu}{2 N}}\left(\int_{\mathbb{R}^{N} \backslash B_{\frac{1}{2 \rho}}} \xi_{\epsilon}(x) d x\right)^{\frac{2 N-\mu}{2 N}} \\
& \leq C \epsilon^{\frac{2 N-\mu}{2}}\left(\int_{\mathbb{R}^{N} \backslash B_{\frac{1}{2 \rho}}} \frac{d x}{|X-(1-\epsilon) \sigma|^{2 N}}\right)^{\frac{2 N-\mu}{2 N}}=O\left(\epsilon^{\frac{2 N-\mu}{2}}\right),
\end{aligned}
$$




$$
\begin{aligned}
J_{4} & \leq C\left(\int_{\mathbb{R}^{N} \backslash B_{\frac{1}{2 \rho}}} \xi_{\epsilon}(x) d x\right)^{\frac{2 N-\mu}{2 N}}\left(\int_{B_{2 \rho}} \xi_{\epsilon}(x) d x\right)^{\frac{2 N-\mu}{2 N}} \\
& \leq C \epsilon^{2 N-\mu}\left(\int_{\mathbb{R}^{N} \backslash B_{\frac{1}{2 \rho}}} \frac{d x}{|x-(1-\epsilon) \sigma|^{2 N}} \int_{B_{2 \rho}} \frac{d x}{|x-(1-\epsilon) \sigma|^{2 N}}\right)^{\frac{2 N-\mu}{2 N}}=O\left(\epsilon^{2 N-\mu}\right), \\
J_{5} & \leq C\left(\int_{\mathbb{R}^{N} \backslash B_{\frac{1}{2 \rho}}} \xi_{\epsilon}(x) d x\right)^{\frac{2 N-\mu}{N}} \leq C \epsilon^{2 N-\mu}\left(\int_{\mathbb{R}^{N \backslash B_{\frac{1}{2 \rho}}}} \frac{d x}{|x-(1-\epsilon) \sigma|^{2 N}}\right)^{\frac{2 N-\mu}{N}} \\
& =O\left(\epsilon^{2 N-\mu}\right) .
\end{aligned}
$$

Therefore, $b\left(g_{\rho}^{\epsilon, \sigma}\right)-\int_{\mathbb{R}^{N}} \int_{\mathbb{R}^{N}} \frac{\left|u_{\epsilon}^{\sigma}(x)\right|^{2_{\mu}^{*}}\left|u_{\epsilon}^{\sigma}(y)\right|^{2_{\mu}^{*}}}{|x-y|^{\mu}} d x d y \rightarrow 0$ as $\epsilon \rightarrow 0$ that is, $b\left(g_{\rho}^{\epsilon, \sigma}\right) \rightarrow S_{H, L}^{\frac{2 N-\mu}{N-\mu+2}}$ as $\epsilon \rightarrow 0$ and completes the proof of (i).

(ii) Result follows from the definition of $\mathcal{J}$ and by (i).

(iii) Assume by contradiction, $g_{\rho}^{\epsilon, \sigma} \rightarrow g_{1} \not \equiv 0$ weakly in $H_{0}^{1}(\Omega)$ then $g_{\rho}^{\epsilon, \sigma} \rightarrow g_{1}$ strongly in $L^{2}(\Omega)$. Then by using the inequality $r^{2(N-2)}+s^{2(N-2)} \leq\left(r^{2}+s^{2}\right)^{N-2}$ for all $r, s \geq 0$, we have

$$
\begin{aligned}
0 \leq \int_{\Omega}\left|g_{\rho}^{\epsilon, \sigma}\right|^{2} d x & \leq C \int_{\frac{3 \rho}{2} \leq|x| \leq \frac{3}{4 \rho}} \frac{\epsilon^{N-2}}{\left(\epsilon^{2}+|x-(1-\epsilon) \sigma|^{2}\right)^{N-2}} d x \\
& =C \int_{\frac{3 \rho}{2} \leq|y+(1-\epsilon) \sigma| \leq \frac{3}{4 \rho}} \frac{\epsilon^{N-2}}{\epsilon^{2(N-2)}+|y|^{2(N-2)}} d y \\
& \leq C \int_{0}^{\frac{3}{4 \rho}+(1-\epsilon)} \frac{\epsilon^{N-2} r^{N-1}}{\epsilon^{2(N-2)}+r^{2(N-2)}} d y \rightarrow 0 .
\end{aligned}
$$

It yields a contradiction. Hence results follows.

Lemma 4.2. Let $\sigma \in \mathbb{S}^{N-1}$ and $\epsilon \in(0,1)$, then the following holds:

(i) $\lim _{\rho \rightarrow 0} \sup _{\sigma \in \mathbb{S}^{N-1}, \epsilon \in(0,1]}\left\|\nabla\left(g_{\rho}^{\epsilon, \sigma}-u_{\epsilon}^{\sigma}\right)\right\|_{L^{2}\left(\mathbb{R}^{N}\right)}^{2}=0$.

(ii) $\lim _{\rho \rightarrow 0} \sup _{\sigma \in \mathbb{S}^{N-1}, \epsilon \in(0,1]}\left\|g_{\rho}^{\epsilon, \sigma}\right\|_{N L}^{2.2_{\mu}^{*}}=\left\|u_{\epsilon}^{\sigma}\right\|_{N L}^{2.2_{\mu}^{*}}$.

\section{Proof.}


(i) Consider

$$
\begin{aligned}
\int_{\mathbb{R}^{N}}\left|\nabla g_{\rho}^{\epsilon, \sigma}-\nabla u_{\epsilon}^{\sigma}\right|^{2} d x \leq & 2 \int_{\mathbb{R}^{N}}\left|u_{\epsilon}^{\sigma}(x) \nabla v_{\rho}(x)\right|^{2} d x+2 \int_{\mathbb{R}^{N}}\left|\nabla u_{\epsilon}^{\sigma}(x) v_{\rho}(x)-\nabla u_{\epsilon}^{\sigma}(x)\right|^{2} d x \\
\leq & C\left(\rho^{-2} \int_{B_{2 \rho}}\left|u_{\epsilon}^{\sigma}(x)\right|^{2} d x+\int_{B_{2 \rho}}\left|\nabla u_{\epsilon}^{\sigma}(x)\right|^{2} d x\right) \\
& +C\left(\rho_{B_{\frac{3}{4 \rho}} \backslash B_{\frac{1}{2 \rho}}}\left|u_{\epsilon}^{\sigma}(x)\right|^{2} d x+\int_{\mathbb{R}^{N} \backslash B_{\frac{1}{2 \rho}}}\left|\nabla u_{\epsilon}^{\sigma}(x)\right|^{2} d x\right) .
\end{aligned}
$$

From the definition of $u_{\varepsilon}^{\sigma}$, we have the following estimates

$$
\begin{gathered}
\rho^{-2} \int_{B_{2 \rho}}\left|u_{\epsilon}^{\sigma}(x)\right|^{2} d x \leq C \rho^{-2} \int_{B_{2 \rho}} d x \leq C \rho^{N-2}, \\
\int_{B_{2 \rho}}\left|\nabla u_{\epsilon}^{\sigma}(x)\right|^{2} d x \leq C \int_{B_{2 \rho}}|x-t \sigma| d x \leq C \int_{B_{2 \rho}} d x \leq C \rho^{N}, \\
\rho^{2} \int_{B_{\frac{3}{4 \rho}} \backslash B_{\frac{1}{2 \rho}}}\left|u_{\epsilon}^{\sigma}(x)\right|^{2} d x \leq C \rho^{2} \int_{B_{\frac{3}{4 \rho}} \backslash B_{\frac{1}{2 \rho}}} \frac{1}{|x|^{2 N-4}} d x \leq C \rho^{N-2}, \\
\int_{\mathbb{R}^{N} \backslash B_{\frac{1}{2 \rho}}}\left|\nabla u_{\epsilon}^{\sigma}(x)\right|^{2} d x \leq C \int_{\mathbb{R}^{N \backslash B_{\frac{1}{2 \rho}}}} \frac{1}{|x|^{2 N-2}} d x \leq C \rho^{N-2} .
\end{gathered}
$$

Therefore, from above estimates and (4.4), we obtain desired result.

(ii) Consider

$$
\begin{aligned}
\left\|g_{\rho}^{\epsilon, \sigma}\right\|_{N L}^{2.22_{\mu}^{*}}-\left\|u_{\epsilon}^{\sigma}\right\|_{N L}^{2.2_{\mu}^{*}} & =\iint_{\mathbb{R}^{N}} \int_{\mathbb{R}^{N}} \frac{\left(v_{\rho}^{2_{\mu}^{*}}(x) v_{\rho}^{2_{\mu}^{*}}(y)-1\right)\left|u_{\epsilon}^{\sigma}(x)\right|^{2_{\mu}^{*}}\left|u_{\epsilon}^{\sigma}(y)\right|^{2_{\mu}^{*}}}{|x-y|^{\mu}} d x d y \\
& \leq C \sum_{i=1}^{5} J_{i},
\end{aligned}
$$

where $J_{i}$ are defined in (4.3). Using the Hardy-Littlewood-Sobolev inequality and the definition of $\xi_{\epsilon}$, we have the following estimates:

$$
\begin{aligned}
J_{1} & \leq C(N, \mu)\left(\int_{B_{2 \rho}} \xi_{\epsilon}(x) d x\right)^{\frac{2 N-\mu}{N}} \leq C\left(\int_{B_{2 \rho}} d x\right)^{\frac{2 N-\mu}{N}} \leq C \rho^{2 N-\mu}, \\
J_{2} & \leq C(N, \mu)\left(\int_{B_{\frac{1}{2 \rho}} \backslash B_{2 \rho}} \xi_{\epsilon}(x) d x\right)^{\frac{2 N-\mu}{2 N}}\left(\int_{B_{2 \rho}} \xi_{\epsilon}(x) d x\right)^{\frac{2 N-\mu}{2 N}} \\
& \leq C\left(\int_{B_{2 \rho}} d x\right)^{\frac{2 N-\mu}{N}} \leq C \rho^{\frac{2 N-\mu}{2}}
\end{aligned}
$$




$$
\begin{aligned}
J_{3} & \leq C(N, \mu)\left(\int_{B_{\frac{1}{2 \rho} \backslash B_{2 \rho}}} \xi_{\epsilon}(x) d x\right)^{\frac{2 N-\mu}{2 N}}\left(\int_{\mathbb{R}^{N} \backslash B_{\frac{1}{2 \rho}}} \xi_{\epsilon}(x) d x\right)^{\frac{2 N-\mu}{2 N}} \\
& \leq C\left(\int_{\mathbb{R}^{N} \backslash B_{\frac{1}{2 \rho}}} \frac{d x}{|x-(1-\epsilon) \sigma|^{2 N}}\right)^{\frac{2 N-\mu}{2 N}} \\
& =\left(\int_{|y+(1-\epsilon) \sigma| \geq \frac{1}{2 \rho}} \frac{\left.d y\right|^{2 N}}{\mid y)^{\frac{2 N-\mu}{2 N}}} \leq\left(\int_{|y| \geq \frac{1}{2 \rho}-1} \frac{d y}{|y|^{2 N}}\right)^{\frac{2 N-\mu}{2 N}} \leq C\left(\frac{(2 \rho)^{N}}{1-(2 \rho)^{N}}\right)^{\frac{2 N-\mu}{2 N}},\right.
\end{aligned}
$$

Now using the same estimates as above we can easily obtain

$$
J_{4} \leq C \rho^{\frac{2 N-\mu}{2}} \text { and } J_{5} \leq C\left(\frac{(2 \rho)^{N}}{1-(2 \rho)^{N}}\right)^{\frac{2 N-\mu}{N}} .
$$

Hence $\sup _{\sigma \in \mathbb{S}^{N-1}, \epsilon \in(0,1]}\left(\left\|g_{\rho}^{\epsilon, \sigma}\right\|_{N L}^{2.2_{\mu}^{*}}-\left\|u_{\epsilon}^{\sigma}\right\|_{N L}^{2.2_{\mu}^{*}}\right) \rightarrow 0$ as $\rho \rightarrow 0$ and completes the proof.

Lemma 4.3. The following asymptic estimates hold:

(i) $a\left(g_{\rho}^{\epsilon, \sigma}\right) \leq S_{H, L}^{\frac{2 N-\mu}{N-\mu+2}}+O\left(\epsilon^{N-2}\right)$.

(ii) $b\left(g_{\rho}^{\epsilon, \sigma}\right) \leq S_{H, L}^{\frac{2 N-\mu}{N-\mu+2}}+O\left(\epsilon^{N}\right)$.

(iii) $b\left(g_{\rho}^{\epsilon, \sigma}\right) \geq S_{H, L}^{\frac{2 N-\mu}{N-\mu+2}}-O\left(\epsilon^{\frac{2 N-\mu}{2}}\right)$.

Proof. Part (i) follows from Lemma 4.1 (i). For part (ii) we will first estimate the integral $\int_{\Omega}\left|g_{\rho}^{\epsilon, \sigma}\right|^{2^{*}} d x$. Since

$$
\int_{\Omega}\left|g_{\rho}^{\epsilon, \sigma}\right|^{2^{*}} d x \leq C \int_{B_{\frac{3}{4 \rho}} \backslash B_{\frac{3 \rho}{2}}}\left|u_{\epsilon}^{\sigma}\right|^{2^{*}} d x \leq \int_{B_{\frac{3}{4}} \backslash B_{\frac{1}{2 \rho}}}\left|u_{\epsilon}^{\sigma}\right|^{2^{*}} d x+\int_{B_{\frac{1}{2 \rho}} \backslash B_{\frac{3 \rho}{2}}}\left|u_{\varepsilon}^{\sigma}\right|^{2^{*}} d x
$$

and

$$
\begin{gathered}
\int_{B_{\frac{3}{4 \rho}} \backslash B_{\frac{1}{2 \rho}}}\left|u_{\epsilon}^{\sigma}\right|^{2^{*}} d x \leq C \epsilon^{N} \int_{B_{\frac{3}{4 \rho}} \backslash B_{\frac{1}{2 \rho}}} \frac{d x}{|x-(1-\epsilon) \sigma|^{2 N}}=O\left(\epsilon^{N}\right), \\
\int_{B_{\frac{1}{2 \rho}} \backslash B_{\frac{3 \rho}{2}}}\left|u_{\epsilon}^{\sigma}\right|^{2^{*}} d x \leq \int_{\mathbb{R}^{N}}\left|u_{\epsilon}^{\sigma}\right|^{2^{*}} d x=S^{\frac{N}{N-\mu+2}} C(N, \mu)^{\frac{-N}{N-\mu+2}} .
\end{gathered}
$$


It implies $\int_{\Omega}\left|g_{\rho}^{\epsilon, \sigma}\right|^{2^{*}} d x \leq S^{\frac{N}{N-\mu+2}} C(N, \mu)^{\frac{-N}{N-\mu+2}}+O\left(\epsilon^{N}\right)$ and now using this and Hardy-Littlewood-Sobolev inequality we have

$$
\begin{aligned}
b\left(g_{\rho}^{\epsilon, \sigma}\right) & =\iint_{\Omega} \frac{\left|g_{\rho}^{\epsilon, \sigma}(x)\right|^{2_{\mu}^{*}}\left|g_{\rho}^{\epsilon, \sigma}(y)\right|^{2_{\mu}^{*}}}{|x-y|^{\mu}} d x d y \\
& \leq C(N, \mu)\left(\int_{\Omega}\left|g_{\rho}^{\epsilon, \sigma}\right|^{2^{*}} d x\right)^{\frac{2 N-\mu}{N}} \\
& \leq C(N, \mu)\left(S^{\frac{N}{N-\mu+2}} C(N, \mu)^{\frac{-N}{N-\mu+2}}+O\left(\epsilon^{N}\right)\right)^{\frac{2 N-\mu}{N}} \leq S_{H, L}^{\frac{2 N-\mu}{N-\mu+2}}+O\left(\epsilon^{N}\right) .
\end{aligned}
$$

This proves part (ii). Now to prove part (iii), consider

$$
\begin{aligned}
& b\left(g_{\rho}^{\epsilon, \sigma}\right)=\int_{\Omega} \int_{\Omega} \frac{\left|g_{\rho}^{\epsilon, \sigma}(x)\right|^{2^{*}}\left|g_{\rho}^{\epsilon, \sigma}(y)\right|^{2_{\mu}^{*}}}{|x-y|^{\mu}} d x d y \\
& \geq \int_{B_{\frac{1}{2 \rho}} \backslash B_{2 \rho}} \int_{\frac{1}{2 \rho} \backslash B_{2 \rho}} \frac{\left|g_{\rho}^{\epsilon, \sigma}(x)\right|^{2_{\mu}^{*}}\left|g_{\rho}^{\epsilon, \sigma}(y)\right|^{2_{\mu}^{*}}}{|x-y|^{\mu}} d x d y \\
& =\iint_{\mathbb{R}^{N}} \frac{\left|u_{\epsilon}^{\sigma}(x)\right|^{2_{\mu}^{*}}\left|u_{\epsilon}^{\sigma}(y)\right|^{2_{\mu}^{*}}}{|x-y|^{\mu}} d x d y-\sum_{i=1}^{i=5} J_{i},
\end{aligned}
$$

where $J_{i}$ are defined in equation (4.3). Using the proof of Lemma 4.1(i) and the fact that $\left\|u_{\varepsilon}^{\sigma}\right\|_{N L}^{2.2_{\mu}^{*}}=S_{H, L}^{\frac{2 N-\mu}{N-\mu+2}}+$ $o_{\epsilon}(1)$, we obtain the required result.

Now we will give a Lemma which is taken from [18]. For the sake of completeness, we provide a complete proof.

Lemma 4.4. If $\mu<\min \{4, N\}$ then

$$
\begin{aligned}
b\left(u_{1}+t g_{\rho}^{\epsilon, \sigma}\right) \geq & b\left(u_{1}\right)+b\left(\operatorname{tg}_{\rho}^{\epsilon, \sigma}\right)+\widehat{C} t^{2.2_{\mu}^{*}-1} \int_{\Omega} \int_{\Omega} \frac{\left(g_{\rho}^{\epsilon, \sigma}(x)\right)^{2_{\mu}^{*}}\left(g_{\rho}^{\epsilon, \sigma}(y)\right)^{2_{\mu}^{*}-1} u_{1}(y)}{|x-y|^{\mu}} d x d y \\
& +2.2_{\mu}^{\star} t \int_{\Omega} \int_{\Omega} \frac{\left(u_{1}(x)\right)^{2_{\mu}^{*}}\left(u_{1}(y)\right)^{2_{\mu}^{*}-1} g_{\rho}^{\epsilon, \sigma}(y)}{|x-y|^{\mu}} d x d y-O\left(\epsilon^{\left(\frac{2 N-\mu}{4}\right) \Theta}\right) \text { for all } \Theta<1,
\end{aligned}
$$

where $u_{1}$ is the local minimum obtained in Lemma 3.10.

Proof. We will divide the proof in two cases:

Case 1: $2_{\mu}^{\star}>3$.

It is easy to see that there exists $\widehat{A}>0$ such that

$$
(a+b)^{p} \geq a^{p}+b^{p}+p a^{p-1} b+\widehat{A} a b^{p-1} \text { for all } a, b \geq 0 \text { and } p>3,
$$

which implies that

$$
\begin{aligned}
b\left(u_{1}+\operatorname{tg}_{\rho}^{\epsilon, \sigma}\right) \geq & b\left(u_{1}\right)+b\left(\operatorname{tg}_{\rho}^{\epsilon, \sigma}\right)+\widehat{C} t^{2.2_{\mu}^{*}-1} \int_{\Omega} \int_{\Omega} \frac{\left(g_{\rho}^{\epsilon, \sigma}(x)\right)^{2_{\mu}^{*}}\left(g_{\rho}^{\epsilon, \sigma}(y)\right)^{2_{\mu}^{*}-1} u_{1}(y)}{|x-y|^{\mu}} d x d y \\
& +2.2_{\mu}^{*} t \int_{\Omega} \int_{\Omega} \frac{\left(u_{1}(x)\right)^{2_{\mu}^{*}}\left(u_{1}(y)\right)^{2_{\mu}^{*}-1} g_{\rho}^{\epsilon, \sigma}(y)}{|x-y|^{\mu}} d x d y, \text { where } \widehat{C}=\min \left\{\widehat{A}, 2.2_{\mu}^{\star}\right\} .
\end{aligned}
$$


Case $2: 2<2_{\mu}^{\star} \leq 3$.

We recall the inequality from [7, Lemma 4]: there exist $C$ (depending on $\left.2_{\mu}^{\star}\right)$ such that, for all $a, b \geq 0$,

$$
(a+b)^{2_{\mu}^{*}} \geq \begin{cases}a^{2_{\mu}^{*}}+b^{2_{\mu}^{*}}+2_{\mu}^{\star} a^{2_{\mu}^{*}-1} b+2_{\mu}^{\star} a b^{2_{\mu}^{*}-1}-C a b^{2_{\mu}^{*}-1} & \text { if } a \geq b, \\ a^{2_{\mu}^{*}}+b^{2_{\mu}^{*}}+2_{\mu}^{\star} a^{2_{\mu}^{*}-1} b+2_{\mu}^{\star} a b^{2_{\mu}^{*}-1}-C a^{2_{\mu}^{*}-1} b & \text { if } a \leq b,\end{cases}
$$

Consider $\Omega \times \Omega=O_{1} \cup O_{2} \cup O_{3} \cup O_{4}$, where

$$
\begin{aligned}
& O_{1}=\left\{(x, y) \in \Omega \times \Omega \mid u_{1}(x) \geq \operatorname{tg}_{\rho}^{\epsilon, \sigma}(x) \text { and } u_{1}(y) \geq \operatorname{tg}_{\rho}^{\epsilon, \sigma}(y)\right\}, \\
& O_{2}=\left\{(x, y) \in \Omega \times \Omega \mid u_{1}(x) \geq \operatorname{tg}_{\rho}^{\epsilon, \sigma}(x) \text { and } u_{1}(y)<\operatorname{tg}_{\rho}^{\epsilon, \sigma}(y)\right\}, \\
& O_{3}=\left\{(x, y) \in \Omega \times \Omega \mid u_{1}(x)<\operatorname{tg}_{\rho}^{\epsilon, \sigma}(x) \text { and } u_{1}(y) \geq \operatorname{tg}_{\rho}^{\epsilon, \sigma}(y)\right\}, \\
& O_{4}=\left\{(x, y) \in \Omega \times \Omega \mid u_{1}(x)<\operatorname{tg}_{\rho}^{\epsilon, \sigma}(x) \text { and } u_{1}(y)<\operatorname{tg}_{\rho}^{\epsilon, \sigma}(y)\right\}
\end{aligned}
$$

Also, define the $b(u)_{\mid O_{i}}=\iint_{O_{i}} \frac{(u(x))^{2_{\mu}^{*}}(u(y))^{2_{\mu}^{*}}}{|x-y|^{\mu}} d x d y$, for all $u \in H_{0}^{1}(\Omega)$ and $i=1,2,3$, 4 .

Subcase 1: when $(x, y) \in O_{1}$.

Employing (4.5), we have the following inequality:

$$
\begin{aligned}
b\left(u_{1}+\operatorname{tg}_{\rho}^{\epsilon, \sigma}\right)_{\mid O_{1}} \geq & \left(b\left(u_{1}\right)+b\left(\operatorname{tg}_{\rho}^{\epsilon, \sigma}\right)\right)_{\mid O_{1}}+2.2_{\mu}^{\star} t^{2.2_{\mu}^{*}-1} \iint_{O_{1}} \frac{\left(g_{\rho}^{\epsilon, \sigma}(x)\right)^{2_{\mu}^{*}}\left(g_{\rho}^{\epsilon, \sigma}(y)\right)^{2_{\mu}^{*}-1} u_{1}(y)}{|x-y|^{\mu}} d x d y \\
& +2.2_{\mu}^{\star} t \iint_{O_{1}} \frac{\left(u_{1}(x)\right)^{2_{\mu}^{*}}\left(u_{1}(y)\right)^{2_{\mu}^{*}-1} g_{\rho}^{\epsilon, \sigma}(y)}{|x-y|^{\mu}} d x d y-A_{\epsilon}^{1},
\end{aligned}
$$

where $A_{\epsilon}^{1}$ is sum of eight non-negative integrals and each integral has an upper bound of the form $C \iint_{O_{1}} \frac{u_{1}(x)\left(\operatorname{tg}_{\rho}^{e, \sigma}(x)\right)^{2^{*} \mu^{-1}}\left(u_{1}(y)\right)^{2^{*}}}{|x-y|^{\mu}} d x d y$ or $C \iint_{O_{1}} \frac{u_{1}(y)\left(\operatorname{tg}_{\rho}^{\epsilon, \sigma}(y)\right)^{2_{\mu}^{*}-1}\left(u_{1}(x)\right)^{2{ }^{*}}}{|x-y|^{\mu}} d x d y$. Write $\left(\operatorname{tg}_{\rho}^{\epsilon, \sigma}(x)\right)^{2^{*} \mu^{-1}}=$ $\left(\operatorname{tg}_{\rho}^{\epsilon, \sigma}(x)\right)^{r} .\left(\operatorname{tg}_{\rho}^{\epsilon, \sigma}(x)\right)^{s}$ with $2_{\mu}^{\star}-1=r+s, 0<s<\frac{2_{\mu}^{*}}{2}$. Then utilizing the definition of $O_{1}, u_{1} \in L^{\infty}(\Omega)$ and Hardy-Littlewood-Sobolev inequality, we have

$$
\begin{aligned}
& \iint_{O_{1}} \frac{u_{1}(x)\left(\operatorname{tg}_{\rho}^{\epsilon, \sigma}(x)\right)^{2_{\mu}^{*}-1}\left(u_{1}(y)\right)^{2_{\mu}^{*}}}{|x-y|^{\mu}} d x d y \leq C \iint_{O_{1}} \frac{\left(u_{1}(x)\right)^{1+r}\left(\operatorname{tg}_{\rho}^{\epsilon, \sigma}(x)\right)^{S}\left(u_{1}(y)\right)^{2_{\mu}^{*}}}{|x-y|^{\mu}} d x d y \\
& \leq C \int_{\Omega} \int_{\Omega} \frac{\left(\operatorname{tg}_{\rho}^{\epsilon, \sigma}(x)\right)^{s}\left(u_{1}(y)\right)^{2_{\mu}^{*}}}{|x-y|^{\mu}} d x d y \\
& \leq C \int_{\Omega} \int_{\Omega} \frac{\epsilon^{\frac{s(N-2)}{2}}}{|x-y|^{\mu}|X-(1-\epsilon) \sigma|^{s(N-2)}} d x d y \\
& \leq C \epsilon^{\frac{s(N-2)}{2}}\left(\int_{\Omega} \frac{d x}{|x-(1-\epsilon) \sigma|^{\frac{s(2 N)(N-2)}{2 N-\mu}}}\right)^{\frac{2 N-\mu}{2 N}} \\
& \leq C \epsilon^{\frac{s(N-2)}{2}}\left(\int_{\Omega} \frac{d x}{|x-(1-\epsilon) \sigma|^{\frac{s(2 N)(N-2)}{2 N-\mu}}}\right)^{\frac{2 N-\mu}{2 N}} .
\end{aligned}
$$

By the choice of $s$ we have $\int_{\Omega} \frac{d x}{|x-(1-\epsilon) \sigma|^{\frac{s(2 N)(N-2)}{2 N-\mu}}}<\infty$. As a result, we get

$$
\iint_{O_{1}} \frac{u_{1}(x)\left(\operatorname{tg}_{\rho}^{\epsilon, \sigma}(x)\right)^{2_{\mu}^{*}-1}\left(u_{1}(y)\right)^{2_{\mu}^{*}}}{|x-y|^{\mu}} d x d y \leq O\left(\epsilon^{\left(\frac{2 N-\mu}{4}\right) \Theta}\right) \text { for all } \Theta<1 \text {. }
$$


In a similar manner, we have

$$
C \iint_{O_{1}} \frac{u_{1}(y)\left(\operatorname{tg}_{\rho}^{\epsilon, \sigma}(y)\right)^{2_{\mu}^{*}-1}\left(u_{1}(x)\right)^{2_{\mu}^{*}}}{|x-y|^{\mu}} d x d y \leq O\left(\epsilon^{\left(\frac{2 N-\mu}{4}\right) \Theta}\right) \text { for all } \Theta<1 .
$$

Subcase 2: when $(x, y) \in O_{2}$.

Once again using (4.5), we have the following inequality:

$$
\begin{aligned}
b\left(u_{1}+\operatorname{tg}_{\rho}^{\epsilon, \sigma}\right)_{\mid O_{2} \geq} \geq & {\left.\left[b\left(u_{1}\right)+b\left(\operatorname{tg}_{\rho}^{\epsilon, \sigma}\right)\right]\right|_{O_{2}}+2.2_{\mu}^{\star} t^{2.2_{\mu}^{*}-1} \iint_{O_{2}} \frac{\left(g_{\rho}^{\epsilon, \sigma}(x)\right)^{2_{\mu}^{*}}\left(g_{\rho}^{\epsilon, \sigma}(y)\right)^{2_{\mu}^{*}-1} u_{1}(y)}{|x-y|^{\mu}} d x d y } \\
& +2.2_{\mu}^{\star} t \iint_{O_{2}} \frac{\left(u_{1}(x)\right)^{2_{\mu}^{*}}\left(u_{1}(y)\right)^{2_{\mu}^{*}-1} g_{\rho}^{\epsilon, \sigma}(y)}{|x-y|^{\mu}} d x d y-A_{\epsilon}^{2},
\end{aligned}
$$

where $A_{\epsilon}^{2}$ is sum of eight non-negative integrals and each integral has an upper bound of the form $C \iint_{O_{2}} \frac{u_{1}(x)\left(\operatorname{tg}_{\rho}^{\epsilon, \sigma}(x)\right)^{2^{*} \mu^{-1}}\left(g_{\rho}^{\epsilon, \sigma}(y)\right)^{2^{*}}}{|x-y|^{\mu}} d x d y$ or $C \iint_{O_{2}} \frac{\left(u_{1}(y)\right)^{2^{*} \mu^{-1}\left(\operatorname{tg}_{\rho}^{\epsilon, \sigma}(y)\right)\left(u_{1}(x)\right)^{2^{*}}}}{|x-y|^{\mu}} d x d y$. By the similar estimates as in Subcase 1 , definition of $O_{2}$, the fact that $\operatorname{tg}_{\rho}^{\epsilon, \sigma} \in H_{0}^{1}(\Omega)$ and regularity of $u_{1}$, we have

$$
\iint_{O_{2}} \frac{u_{1}(x)\left(\operatorname{tg}_{\rho}^{\epsilon, \sigma}(x)\right)^{2_{\mu}^{*}-1}\left(g_{\rho}^{\epsilon, \sigma}(y)\right)^{2_{\mu}^{*}}}{|x-y|^{\mu}} d x d y \leq O\left(\epsilon^{\left(\frac{2 N-\mu}{4}\right) \Theta}\right) \text { for all } \Theta<1 .
$$

Write $\left(u_{1}(y)\right)^{2_{\mu}^{*}-1}=\left(u_{1}(y)\right)^{r} .\left(u_{1}(y)\right)^{s}$ with $2_{\mu}^{*}-1=r+s, 0<1+s<\frac{2_{\mu}^{*}}{2}$. Then utilizing the definition of $O_{2}$, $u_{1} \in L^{\infty}(\Omega)$ and Hardy-Littlewood-Sobolev inequality, we have

$$
\begin{aligned}
\iint_{O_{2}} \frac{\left(u_{1}(y)\right)^{2_{\mu}^{*}-1}\left(\operatorname{tg}_{\rho}^{\epsilon, \sigma}(y)\right)\left(u_{1}(x)\right)^{2_{\mu}^{*}}}{|x-y|^{\mu}} & d x d y \leq \iint_{O_{2}} \frac{\left(u_{1}(y)\right)^{r}\left(\operatorname{tg}_{\rho}^{\epsilon, \sigma}(y)\right)^{1+s}\left(u_{1}(x)\right)^{2_{\mu}^{*}}}{|x-y|^{\mu}} d x d y \\
& \leq C \iint_{\Omega} \frac{\left(\operatorname{tg}_{\rho}^{\epsilon, \sigma}(y)\right)^{1+s}\left(u_{1}(x)\right)^{2_{\mu}^{*}}}{|x-y|^{\mu}} d x d y \\
& \leq C \int_{\Omega} \int_{\Omega} \frac{\epsilon^{\frac{(1+s)(N-2)}{2}}}{|x-y|^{\mu}|y-(1-\epsilon) \sigma|^{(1+s)(N-2)}} d x d y \\
& \leq C \epsilon^{\frac{(1+s)(N-2)}{2}}\left(\int_{\Omega} \frac{d y}{|y-(1-\epsilon) \sigma|^{\frac{(1+s)(2 N)(N-2)}{2 N-\mu}}}\right)^{\frac{2 N-\mu}{2 N}} \\
& \leq C \epsilon^{\frac{(1+s)(N-2)}{2}}\left(\int_{\Omega} \frac{d y}{|y-(1-\epsilon) \sigma|^{\frac{(1+s)(2 N)(N-2)}{2 N-\mu}}}\right)^{\frac{2 N-\mu}{2 N}} .
\end{aligned}
$$

By the choice of $s$ we have $\int_{\Omega} \frac{d x}{|x-(1-\epsilon) \sigma|^{\frac{(1+s)(2 N)(N-2)}{2 N-\mu}}}<\infty$. Hence we obtain

$$
\iint_{O_{2}} \frac{\left(u_{1}(y)\right)^{2_{\mu}^{\star}-1}\left(\operatorname{tg}_{\rho}^{\epsilon, \sigma}(y)\right)\left(u_{1}(x)\right)^{2_{\mu}^{\star}}}{|x-y|^{\mu}} d x d y \leq O\left(\epsilon^{\left(\frac{2 N-\mu}{4}\right) \Theta}\right) \text { for all } \Theta<1 .
$$


Subcase 3: when $(x, y) \in O_{3}$.

Using (4.5), we have

$$
\begin{aligned}
b\left(u_{1}+\operatorname{tg}_{\rho}^{\epsilon, \sigma}\right)_{\mid O_{3}} \geq & \left.\left(b\left(u_{1}\right)+b\left(\operatorname{tg}_{\rho}^{\epsilon, \sigma}\right)\right)\right|_{O_{3}}+2.2_{\mu}^{*} t^{2.2_{\mu}^{*}-1} \iint_{O_{3}} \frac{\left(g_{\rho}^{\epsilon, \sigma}(x)\right)^{2_{\mu}^{*}}\left(g_{\rho}^{\epsilon, \sigma}(y)\right)^{2_{\mu}^{*}-1} u_{1}(y)}{|x-y|^{\mu}} d x d y \\
& +2.2_{\mu}^{\star} t \iint_{O_{3}} \frac{\left(u_{1}(x)\right)^{2_{\mu}^{*}}\left(u_{1}(y)\right)^{2_{\mu}^{*}-1} g_{\rho}^{\epsilon, \sigma}(y)}{|x-y|^{\mu}} d x d y-A_{\epsilon}^{3},
\end{aligned}
$$

where $A_{\epsilon}^{3}$ is sum of eight non-negative integrals and each integral has an upper bound of the form $C \iint_{O_{3}} \frac{\left(u_{1}(x)\right)^{2^{*} \mu^{-1}}\left(\operatorname{tg}_{\rho}^{\epsilon, \sigma}(x)\right)\left(u_{1}(y)\right)^{2^{*} \mu}}{|x-y|^{\mid}} d x d y$ or $C \iint_{O_{3}} \frac{u_{1}(y)\left(t g_{\rho}^{\epsilon, \sigma}(y)\right)^{*^{*}-1}\left(g_{\rho}^{e, \sigma}(x)\right)^{2^{*} \mu}}{|x-y|^{\mu}} d x d y$. By the similar estimates as in Subcase 1, Subcase 2, definition of $O_{3}$ and regularity of $u_{1}$, we get $A_{\epsilon}^{3} \leq O\left(\epsilon^{\left(\frac{2 N-\mu}{4}\right) \Theta}\right)$ for all $\Theta<1$.

Subcase 4: when $(x, y) \in O_{4}$.

Using (4.5), we have

$$
\begin{aligned}
b\left(u_{1}+\operatorname{tg}_{\rho}^{\epsilon, \sigma}\right)_{\mid O_{4}} \geq & \left.\left(b\left(u_{1}\right)+b\left(\operatorname{tg}_{\rho}^{\epsilon, \sigma}\right)\right)\right|_{O_{4}}+2.2_{\mu}^{*} t^{2.2_{\mu}^{*}-1} \iint_{O_{4}} \frac{\left(g_{\rho}^{\epsilon, \sigma}(x)\right)^{2_{\mu}^{*}}\left(g_{\rho}^{\epsilon, \sigma}(y)\right)^{2_{\mu}^{*}-1} u_{1}(y)}{|x-y|^{\mu}} d x d y \\
& +2.2_{\mu}^{*} t \iint_{O_{4}} \frac{\left(u_{1}(x)\right)^{2_{\mu}^{*}}\left(u_{1}(y)\right)^{2_{\mu}^{*}-1} g_{\rho}^{\epsilon, \sigma}(y)}{|x-y|^{\mu}} d x d y-A_{\epsilon}^{4},
\end{aligned}
$$

where $A_{\epsilon}^{4}$ is sum of eight non-negative integrals and each integral has an upper bound of the form $C \iint_{O_{4}} \frac{\left(u_{1}(x)\right)^{2^{*}-1}\left(\operatorname{tg}_{\rho}^{\epsilon, \sigma}(x)\right)\left(\operatorname{tg}_{\rho}^{\boldsymbol{g}, \sigma}(y)\right)^{2_{\mu}^{*}}}{|x-y|^{\mu}} d x d y$ or $C \iint_{O_{4}} \frac{u_{1}(y)\left(\operatorname{tg}_{\rho}^{g, \sigma}(y)\right)^{2_{\mu}^{*}-1}\left(g_{\rho}^{\epsilon, \sigma}(x)\right)^{2_{\mu}^{*}}}{|x-y|^{\mu}} d x d y$. By the similar estimates as in Subcase 2, we have

$$
A_{\epsilon}^{4} \leq O\left(\epsilon^{\left(\frac{2 N-\mu}{4}\right) \Theta}\right) \text { for all } \Theta<1 .
$$

From all subcases we obtain $A_{\epsilon}^{i} \leq O\left(\epsilon^{\left(\frac{2 N-\mu}{4}\right) \Theta}\right)$ for all $\Theta<1$ and $i=1,2,3,4$. Combining all sub cases we conclude Case 2. From Case 1 and Case 2 we have the required result.

Proposition 4.5. Let $\mu<\min \{4, N\}$ then there exists $\epsilon_{0}>0$ such that for every $0<\epsilon<\epsilon_{0}$ we have

$$
\sup _{t \geq 0} \mathcal{J}_{f}\left(u_{1}+\operatorname{tg}_{\rho}^{\epsilon, \sigma}\right)<\mathcal{J}_{f}\left(u_{1}\right)+\frac{N-\mu+2}{2(2 N-\mu)} S_{H, L}^{\frac{2 N-\mu}{N-2}} \text { uniformly in } \sigma \in \mathbb{S}^{N-1},
$$

where $u_{1}$ is the local minimum in Lemma 3.10.

Proof. By Lemma 3.8, $u \in L^{\infty}(\Omega)$ and $u>0$ in $\Omega$. This implies

$$
b\left(u_{1}+\operatorname{tg}_{\rho}^{\epsilon, \sigma}\right)=\int_{\Omega} \int_{\Omega} \frac{\left(u_{1}+\operatorname{tg}_{\rho}^{\epsilon, \sigma}(x)\right)^{2_{\mu}^{*}}\left(u_{1}+\operatorname{tg}_{\rho}^{\epsilon, \sigma}(y)\right)^{2_{\mu}^{*}}}{|x-y|^{\mu}} d x d y .
$$

Claim 1: There exists a $R_{0}>0$ such that

$$
I=\iint_{\Omega} \frac{\left(g_{\rho}^{\epsilon, \sigma}(x)\right)^{2^{*}}\left(g_{\rho}^{\epsilon, \sigma}(y)\right)^{2_{\mu}^{*}-1} u_{1}(y)}{|x-y|^{\mu}} d x d y \geq \widehat{C} R_{0} \epsilon^{\frac{N-2}{2}} .
$$


Clearly,

$$
\begin{aligned}
I \geq & \int_{B_{\frac{1}{2 \rho}} \backslash B_{2 \rho} B_{\frac{1}{2 \rho}}} \int_{B_{2 \rho}} \frac{\left(g_{\rho}^{\epsilon, \sigma}(x)\right)^{2_{\mu}^{*}}\left(g_{\rho}^{\epsilon, \sigma}(y)\right)^{2_{\mu}^{*}-1} u_{1}(y)}{|x-y|^{\mu}} d x d y \\
& \geq C \int_{B_{\frac{1}{2 \rho}} \backslash B_{2 \rho} \rho} \int_{\frac{1}{2 \rho} \backslash B_{2 \rho}} \frac{\left(u_{\epsilon}^{\sigma}(x)\right)^{2_{\mu}^{*}}\left(u_{\epsilon}^{\sigma}(y)\right)^{2_{\mu}^{*}-1}}{|x-y|^{\mu}} d x d y \\
& \geq C \int_{B_{\frac{1}{2 \rho}} \backslash B_{2 \rho}} \int_{\frac{1}{2 \rho} \backslash B_{2 \rho}} \frac{\epsilon^{\frac{3 N}{2}+1-\mu} d x d y}{|x-y|^{\mu}\left(\epsilon^{2}+|x-(1-\epsilon) \sigma|^{2}\right)^{\frac{2 N-\mu}{2}}\left(\epsilon^{2}+|y-(1-\epsilon) \sigma|^{2}\right)^{\frac{N-\mu+2}{2}}} .
\end{aligned}
$$

For any $\epsilon<1-2 \rho$ there exists $c>0$ such that $1-\epsilon>c>2 \rho$ so we get

$$
\begin{aligned}
I & \geq C \epsilon^{\frac{3 N}{2}+1-\mu} \int_{B_{c}} \int_{B_{c}} \frac{d z d w}{|z-w|^{\mu}\left(\epsilon^{2}+|z|^{2}\right)^{\frac{2 N-\mu}{2}}\left(\epsilon^{2}+|w|^{2}\right)^{\frac{N-\mu+2}{2}}} \\
& \geq C \epsilon^{\frac{N-2}{2}} \int_{B_{C}} \int_{B_{C}} \frac{d z d w}{|z-w|^{\mu}\left(1+|z|^{2}\right)^{\frac{2 N-\mu}{2}}\left(1+|w|^{2}\right)^{\frac{N-\mu+2}{2}}}=O\left(\epsilon^{\frac{N-2}{2}}\right) .
\end{aligned}
$$

This proves the claim 1. Now using Lemma 4.4, we have

$$
\begin{aligned}
\partial_{f}\left(u_{1}+\operatorname{tg}_{\rho}^{\epsilon, \sigma}\right) \leq & \frac{1}{2} a\left(u_{1}\right)+\frac{1}{2} a\left(\operatorname{tg}_{\rho}^{\epsilon, \sigma}\right)+t\left\langle u_{1}, g_{\rho}^{\epsilon, \sigma}\right\rangle_{H_{0}^{1}(\Omega)}-\frac{1}{2.2_{\mu}^{\star}} b\left(u_{1}\right)-\frac{1}{2.2_{\mu}^{\star}} b\left(\operatorname{tg}_{\rho}^{\epsilon, \sigma}\right) \\
& -\widehat{C} t^{2.2_{\mu}^{*}-1} \int_{\Omega} \int_{\Omega} \frac{\left(g_{\rho}^{\epsilon, \sigma}(x)\right)^{2_{\mu}^{*}}\left(g_{\rho}^{\epsilon, \sigma}(y)\right)^{2_{\mu}^{*}-1} u_{1}(y)}{|x-y|^{\mu}} d x d y-\int_{\Omega} f u_{1} d x \\
& -t \int_{\Omega} f g_{\rho}^{\epsilon, \sigma} d x-t \int_{\Omega} \int_{\Omega} \frac{\left(u_{1}(x)\right)^{2_{\mu}^{*}}\left(u_{1}(y)\right)^{2_{\mu}^{\star}-1} g_{\rho}^{\epsilon, \sigma}(y)}{|x-y|^{\mu}} d x d y+O\left(\epsilon^{\left(\frac{2 N-\mu}{4}\right) \Theta}\right) .
\end{aligned}
$$

for all $\Theta<1$. Taking $\Theta=\frac{2}{2_{\mu}^{*}}$, we have

$$
\begin{aligned}
\partial_{f}\left(u_{1}+\operatorname{tg}_{\rho}^{\epsilon, \sigma}\right) \leq & \frac{1}{2} a\left(u_{1}\right)+\frac{1}{2} a\left(\operatorname{tg}_{\rho}^{\epsilon, \sigma}\right)+t\left\langle u_{1}, g_{\rho}^{\epsilon, \sigma}\right\rangle_{H_{0}^{1}(\Omega)}-\frac{1}{2.2_{\mu}^{\star}} b\left(u_{1}\right)-\frac{1}{2.2_{\mu}^{\star}} b\left(\operatorname{tg}_{\rho}^{\epsilon, \sigma}\right) \\
& -\widehat{C} t^{2.2_{\mu^{*}}^{*}-1} \int_{\Omega} \int_{\Omega} \frac{\left(g_{\rho}^{\epsilon, \sigma}(x)\right)^{2_{\mu}^{*}}\left(g_{\rho}^{\epsilon, \sigma}(y)\right)^{2_{\mu}^{*}-1} u_{1}(y)}{|x-y|^{\mu}} d x d y-\int_{\Omega} f u_{1} d x \\
& -t \int_{\Omega} f g_{\rho}^{\epsilon, \sigma} d x-t \int_{\Omega} \int_{\Omega} \frac{\left(u_{1}(x)\right)^{2_{\mu}^{*}}\left(u_{1}(y)\right)^{2_{\mu}^{*}-1} g_{\rho}^{\epsilon, \sigma}(y)}{|x-y|^{\mu}} d x d y+o\left(\epsilon^{\frac{N-2}{2}}\right) .
\end{aligned}
$$


This on utilizing Lemma 4.3 and claim 1 gives

$$
\begin{aligned}
\partial_{f}\left(u_{1}+\operatorname{tg}_{\rho}^{\epsilon, \sigma}\right) \leq & \frac{1}{2} a\left(u_{1}\right)+\frac{1}{2} a\left(\operatorname{tg}_{\rho}^{\epsilon, \sigma}\right)+t\left\langle u_{1}, g_{\rho}^{\epsilon, \sigma}\right\rangle_{H_{0}^{1}(\Omega)}-\frac{1}{2.2_{\mu}^{\star}} b\left(u_{1}\right)-\frac{1}{2.2_{\mu}^{\star}} b\left(\operatorname{tg}_{\rho}^{\epsilon, \sigma}\right) \\
& -\widehat{C} t^{2.2_{\mu}^{*}-1} \int_{\Omega} \int_{\Omega} \frac{\left(g_{\rho}^{\epsilon, \sigma}(x)\right)^{2_{\mu}^{*}}\left(g_{\rho}^{\epsilon, \sigma}(y)\right)^{2_{\mu}^{*}-1}}{|x-y|^{\mu}} d x d y-\int_{\Omega} f u_{1} d x-t \int_{\Omega} f g_{\rho}^{\epsilon, \sigma} d x \\
& -t \int_{\Omega} \frac{\left(u_{1}(x)\right)^{2_{\mu}^{*}}\left(u_{1}(y)\right)^{2_{\mu}^{*}-1} g_{\rho}^{\epsilon, \sigma}(y)}{|x-y|^{\mu}} d x d y+o\left(\epsilon^{\frac{N-2}{2}}\right) \\
= & \partial_{f}\left(u_{1}\right)+\partial\left(t g_{\rho}^{\epsilon, \sigma}\right)-\widehat{C} t^{2.2_{\mu}^{*}-1} \iint_{\Omega} \frac{\left(g_{\rho}^{\epsilon, \sigma}(x)\right)^{2_{\mu}^{*}}\left(g_{\rho}^{\epsilon, \sigma}(y)\right)^{2_{\mu}^{*}-1}}{|x-y|^{\mu}} d x d y+o\left(\epsilon^{\frac{N-2}{2}}\right) \\
\leq & \partial_{f}\left(u_{1}\right)+\frac{t^{2}}{2}\left(S_{H, L}^{\frac{2 N-\mu}{N-\mu+2}}+O\left(\epsilon^{N-2}\right)\right)-\frac{t^{2.2_{\mu}^{*}}}{2.2_{\mu}^{\star}}\left(S_{H, L}^{\frac{2 N-\mu}{N-\mu+2}}-O\left(\epsilon^{\frac{2 N-\mu}{2}}\right)\right) \\
& -t^{2.2_{\mu}^{*}-1} \widehat{C} R_{0} \epsilon^{\frac{N-2}{2}}+o\left(\epsilon^{\frac{N-2}{2}}\right) .
\end{aligned}
$$

Now define $K(t):=\frac{t^{2}}{2}\left(S_{H, L}^{\frac{2 N-\mu}{N-\mu+2}}+O\left(\epsilon^{N-2}\right)\right)-\frac{t^{2.2_{\mu}^{\star}}}{2 \cdot 2_{\mu}^{\star}}\left(S_{H, L}^{\frac{2 N-\mu}{N-\mu+2}}-O\left(\epsilon^{\frac{2 N-\mu}{2}}\right)\right)-t^{2.2_{\mu}^{\star}-1} \widehat{C} R_{0} \epsilon^{\frac{N-2}{2}}$ then $K(t) \rightarrow \infty$ as $t \rightarrow$

$\infty$ and $\lim _{t \rightarrow 0^{+}} K(t)>0$ so there exists a $t_{\epsilon}>0$ such that $\sup _{t>0} K(t)$ is attained and $t_{\epsilon}<\left(\frac{S_{H, L}^{\frac{2 N-\mu}{N-\mu}}+O\left(\epsilon^{N-2}\right)}{S_{H, L}^{\frac{N N-\mu}{N-\mu+2}}-O\left(\epsilon^{\frac{2 N-\mu}{2}}\right)}\right)^{\frac{1}{2.2 \mu^{*}-2}}:=$ $S_{H, L}(\epsilon)$. Moreover there exists a $t_{1}>0$ such that for sufficiently small $\epsilon>0$ we have $t_{\epsilon}>t_{1}$. Clearly the function

$$
t \mapsto \frac{t^{2}}{2}\left(S_{H, L}^{\frac{2 N-\mu}{N-\mu+2}}+O\left(\epsilon^{N-2}\right)\right)-\frac{t^{2 \cdot 2_{\mu}^{*}}}{2.2_{\mu}^{\star}}\left(S_{H, L}^{\frac{2 N-\mu}{N-\mu+2}}-O\left(\epsilon^{\frac{2 N-\mu}{2}}\right)\right)
$$

is an increasing function in $\left[0, S_{H, L}(\epsilon)\right]$. Therefore,

$$
\begin{aligned}
\sup _{t \geq 0} \mathcal{J}_{f}\left(u_{1}+t g_{\rho}^{\epsilon, \sigma}\right) \leq & \partial_{f}\left(u_{1}\right)+\frac{N-\mu+2}{2(2 N-\mu)} S_{H, L}^{\frac{2 N-\mu}{N-\mu+2}}+O\left(\epsilon^{\min \left\{\frac{2 N-\mu}{2}, N-2\right\}}\right) \\
& -t_{1}^{2.2_{\mu}^{*}-1} \widehat{C} R_{0} \epsilon^{\frac{N-2}{2}}+o\left(\epsilon^{\frac{N-2}{2}}\right) .
\end{aligned}
$$

Hence there exits a $\epsilon_{0}>0$ such that for every $0<\epsilon<\epsilon_{0}$ we have

$$
\sup _{t \geq 0} \partial_{f}\left(u_{1}+g_{\rho}^{\epsilon, \sigma}\right)<\partial_{f}\left(u_{1}\right)+\frac{N-\mu+2}{2(2 N-\mu)} S_{H, L}^{\frac{2 N-\mu}{N-\mu+2}} \text { uniformly in } \sigma \in \mathbb{S}^{N-1} .
$$

Lemma 4.6. The following holds:

(i) $H_{0}^{1}(\Omega) \backslash \mathcal{N}_{f}^{-}=U_{1} \cup U_{2}$, where

$$
\begin{aligned}
& U_{1}:=\left\{u \in H_{0}^{1}(\Omega) \backslash\{0\} \mid u^{+} \not \equiv 0,\|u\|<t^{-}\left(\frac{u}{\|u\|}\right)\right\} \cup\{0\}, \\
& U_{2}:=\left\{u \in H_{0}^{1}(\Omega) \backslash\{0\} \mid u^{+} \not \equiv 0,\|u\|>t^{-}\left(\frac{u}{\|u\|}\right)\right\} .
\end{aligned}
$$

(ii) $\mathcal{N}_{f}^{+} \subset U_{1}$.

(iii) For each $0<\epsilon \leq \epsilon_{0}$, there exists $t_{0}>1$ and such that $u_{1}+t_{0} g_{\rho}^{\epsilon, \sigma} \in U_{2}$.

(iv) For each $0<\epsilon<\epsilon_{0}$, there exists $s_{0} \subset(0,1)$ and such that $u_{1}+s_{0} t_{0} g_{\rho}^{\epsilon, \sigma} \in \mathcal{N}_{f}^{-}$. 
(v) $\Upsilon_{f}^{-}<\Upsilon_{f}+\frac{N-\mu+2}{2(2 N-\mu)} S_{H, L}^{\frac{2 N-\mu}{N-\mu+2}}$.

\section{Proof.}

(i) It holds by Lemma $3.3(\mathrm{~d})$.

(ii) Let $u \in \mathcal{N}_{f}^{+}$then $t^{+}(u)=1$ and $1<t^{+}(u)<t_{\max }<t^{-}(u)=\frac{1}{\|u\|} t^{-}\left(\frac{u}{\|u\|}\right)$ that is, $\mathcal{N}_{f}^{+} \subset U_{1}$.

(iii) First, we will show that there exists a constant $c>0$ such that $0<t^{-}\left(\frac{u_{1}+t_{\rho}^{\varepsilon, \sigma}}{\left\|u_{1}+t_{\rho}^{\epsilon, \sigma}\right\|}\right)<c$ for all $t>0$. On the contrary, let there exist a sequence $\left\{t_{n}\right\}$ such that $t_{n} \rightarrow \infty$ and $t^{-}\left(\frac{u_{1}+t_{n} g_{\rho}^{, \sigma o}}{\left\|u_{1}+t_{n} g_{\rho}^{, \sigma}\right\|}\right) \rightarrow \infty$ as $n \rightarrow \infty$. Define $u_{n}:=\frac{u_{1}+t_{n} g_{\rho}^{e, \sigma}}{\left\|u_{1}+t_{n} g_{\rho}^{\varepsilon, \sigma}\right\|}$ so there exists $t^{-}\left(u_{n}\right)$ such that $t^{-}\left(u_{n}\right) u_{n} \in \mathcal{N}_{f}^{-}$. By dominated convergence theorem,

$$
b\left(u_{n}\right)=\frac{b\left(u_{1}+t_{n} g_{\rho}^{\epsilon, \sigma}\right)}{\left\|u_{1}+t_{n} g_{\rho}^{\epsilon, \sigma}\right\|^{2.2_{\mu}^{*}}}=\frac{b\left(\frac{u_{1}}{t_{n}}+g_{\rho}^{\epsilon, \sigma}\right)}{\left\|\frac{u_{1}}{t_{n}}+g_{\rho}^{\epsilon, \sigma}\right\|^{2.2_{\mu}^{*}}} \rightarrow \frac{b\left(g_{\rho}^{\epsilon, \sigma}\right)}{\left\|g_{\rho}^{\epsilon, \sigma}\right\|^{2.2_{\mu}^{*}}} \text { as } n \rightarrow \infty .
$$

Hence, $\partial_{f}\left(t^{-}\left(u_{n}\right) u_{n}\right) \rightarrow-\infty$ as $n \rightarrow \infty$, contradicts the fact that $\mathcal{J}_{f}$ is bounded below on $\mathcal{N}_{f}$. Therefore, there exists $c>0$ such that $0<t^{-}\left(\frac{u_{1}+\operatorname{tg}_{\rho}^{\epsilon, \sigma}}{\left\|u_{1}+\operatorname{tg}_{\rho}^{\epsilon, \sigma}\right\|}\right)<c$ for all $t>0$. Let $t_{0}=\frac{\left|c^{2}-\left\|u_{1}\right\|^{2}\right|^{\frac{1}{2}}}{\left\|g_{\rho}^{\epsilon, \sigma}\right\|}+1$ then

$$
\begin{aligned}
\left\|u_{1}+t_{0} g_{\rho}^{\epsilon, \sigma}\right\|^{2} & =\left\|u_{1}\right\|^{2}+t_{0}^{2}\left\|g_{\rho}^{\epsilon, \sigma}\right\|^{2}+2 t_{0}\left\langle u_{1}, g_{\rho}^{\epsilon, \sigma}\right\rangle \\
& \geq\left\|u_{1}\right\|^{2}+\left|c^{2}-\left\|u_{1}\right\|^{2}\right| \geq c^{2} \geq\left(t^{-}\left(\frac{u_{1}+g_{\rho}^{\epsilon, \sigma}}{\left\|u_{1}+\operatorname{tg}_{\rho}^{\epsilon, \sigma}\right\|}\right)\right)^{2} .
\end{aligned}
$$

It implies that $u_{1}+t_{0} g_{\rho}^{\epsilon, \sigma} \in U_{2}$.

(iv) For each $0<\epsilon<\epsilon_{0}$, define a path $\xi_{\epsilon}(s)=u_{1}+s t_{0} g_{\rho}^{\epsilon, \sigma}$ for $s \in[0,1]$. Then

$$
\xi_{\epsilon}(0)=u_{1} \quad \text { and } \quad \xi_{\epsilon}(1)=u_{1}+t_{0} g_{\rho}^{\epsilon, \sigma} \in U_{2} .
$$

Since $\frac{1}{\|u\|} t^{-}\left(\frac{u}{\|u\|}\right)$ is a continuous function and $\xi_{\epsilon}([0,1])$ is connected. So, there exists $s_{0} \in[0,1]$ such that $\xi_{\epsilon}\left(s_{0}\right)=u_{1}+s_{0} t_{0} g_{\rho}^{\epsilon, \sigma} \in \mathcal{N}_{f}^{-}$.

(v) Using part (iv) and Proposition 4.5.

At this point we will state Global compactness Lemma for the functional $\mathcal{J}_{f}$ which is a version of Theorem 4.4 of [19].

Lemma 4.7. Let $\left\{u_{n}\right\} \subset H_{0}^{1}(\Omega)$ be such that $\mathcal{J}_{f}\left(u_{n}\right) \rightarrow c, \partial_{f}^{\prime}\left(u_{n}\right) \rightarrow 0$. Then passing if necessary to a subsequence, there exists a solution $v_{0} \in H_{0}^{1}(\Omega)$ of

$$
-\Delta u=\left(\int_{\Omega} \frac{\left|u^{+}(y)\right|^{2_{\mu}^{*}}}{|x-y|^{\mu}} d y\right)\left|u^{+}\right|^{2_{\mu}^{*}-1}+f \text { in } \Omega
$$

and (possibly) $k \in \mathbb{N} \cup\{0\}$, non-trivial solutions $\left\{v_{1}, v_{2}, \ldots, v_{k}\right\}$ of

$$
-\Delta u=\left(|x|^{-\mu \star}\left|u^{+}\right|^{2_{\mu}^{*}}\right)\left|u^{+}\right|^{2_{\mu}^{*}-1} \text { in } \mathbb{R}^{N}
$$

with $v_{i} \in D^{1,2}\left(\mathbb{R}^{N}\right)$ and $k$ sequences $\left\{y_{n}^{i}\right\}_{n} \subset \Omega$ and $\left\{\lambda_{n}^{i}\right\}_{n} \subset \mathbb{R}_{+} i=1,2, \cdots k$, satisfying

$$
\begin{gathered}
\frac{1}{\lambda_{n}^{i}} \operatorname{dist}\left(y_{n}^{i}, \partial \Omega\right) \rightarrow \infty, \text { and }\left\|u_{n}-v_{0}-\sum_{i=1}^{k}\left(\lambda_{n}^{i}\right)^{\frac{2-N}{2}} v_{i}\left(\left(.-y_{n}^{i}\right) / \lambda_{n}^{i}\right)\right\|_{D^{1,2}\left(\mathbb{R}^{N}\right)} \rightarrow 0, n \rightarrow \infty, \\
\left\|u_{n}\right\|_{D^{1,2}\left(\mathbb{R}^{N}\right)}^{2} \rightarrow \sum_{i=0}^{k}\left\|v_{i}\right\|_{D^{1,2}\left(\mathbb{R}^{N}\right)}^{2}, \text { as } n \rightarrow \infty, \quad \partial_{f}\left(v_{0}\right)+\sum_{i=1}^{k} \partial_{\infty}\left(v_{i}\right)=c,
\end{gathered}
$$

where $J_{\infty}(u):=\frac{1}{2} \int_{\mathbb{R}^{N}}|\nabla u|^{2} d x-\frac{1}{2.2_{\mu}^{*}} \int_{\mathbb{R}^{N}} \int_{\mathbb{R}^{N}} \frac{\left|u^{+}(x)\right|^{2_{\mu}^{*}}\left|u^{+}(y)\right|^{2_{\mu}^{*}}}{|x-y|^{\mu}} d x d y, \quad u \in D^{1,2}\left(\mathbb{R}^{N}\right)$. 
Lemma 4.8. (i) Let $\left\{u_{n}\right\}$ be a $(P S)_{c}$ sequence for $\partial_{f}$ with $c<\Upsilon_{f}(\Omega)+\frac{N-\mu+2}{2(2 N-\mu)} S_{H, L}^{\frac{2 N-\mu}{N-\mu+2}}$ then there exists a subsequence still denoted by $\left\{u_{n}\right\}$ and a nonzero $u^{0} \in H_{0}^{1}(\Omega)$ such that $u_{n} \rightarrow u^{0}$ strongly in $H_{0}^{1}(\Omega)$ and $\mathcal{J}_{f}\left(u^{0}\right)=c$.

(ii) Let $\left\{u_{n}\right\} \subset \mathcal{N}_{f}^{-}$be a $(P S)_{c}$ sequence for $\mathcal{J}_{f}$ with

$$
\Upsilon_{f}(\Omega)+\frac{N-\mu+2}{2(2 N-\mu)} S_{H, L}^{\frac{2 N-\mu}{N-\mu+2}}<c<\Upsilon_{f}^{-}(\Omega)+\frac{N-\mu+2}{2(2 N-\mu)} S_{H, L}^{\frac{2 N-\mu}{N-\mu+2}}
$$

then there exists subsequence still denoted by $\left\{u_{n}\right\}$ and a nonzero $u^{0} \in \mathcal{N}_{f}^{-}$such that $u_{n} \rightarrow u^{0}$ strongly in $H_{0}^{1}(\Omega)$ and $\mathcal{J}_{f}\left(u^{0}\right)=c$.

Proof. Proof of (i) follows from [30, Lemma 3.4]. To prove (ii), Let $\left\{u_{n}\right\}$ be a $(P S)_{c}$ sequence then by standard arguments $\left\{u_{n}\right\}$ is bounded in $H_{0}^{1}(\Omega)$ and there exists a subsequence of $\left\{u_{n}\right\}$ still denoted by $\left\{u_{n}\right\}$ and $u^{0} \in$ $H_{0}^{1}(\Omega)$ such that $u_{n} \rightarrow u^{0}$ in $H_{0}^{1}(\Omega)$ and $\mathcal{J}_{f}^{\prime}\left(u^{0}\right)=0$. Then by Lemma 3.11, we have either $u^{0} \in \mathcal{N}_{f}^{-}$or $u^{0}=u_{1}$. Now using Lemma 4.7 we obtain

$$
\Upsilon_{f}^{-}(\Omega)+\frac{N-\mu+2}{2(2 N-\mu)} S_{H, L}^{\frac{2 N-\mu}{N-\mu+2}} \geq c=\partial_{f}\left(u^{0}\right)+\sum_{i=1}^{k} \partial_{\infty}\left(v_{i}\right) \geq \Upsilon_{f}(\Omega)+k \frac{N-\mu+2}{2(2 N-\mu)} S_{H, L}^{\frac{2 N-\mu}{N-\mu+2}} .
$$

which on using Lemma 4.6(e), gives $k \leq 1$. By [19, corollary 3.3], we get $v_{1}$ is a constant multiple of Talenti function that is, $J_{\infty}\left(v_{1}\right)=\frac{N-\mu+2}{2(2 N-\mu)} S_{H, L}^{\frac{2 N-\mu}{N-\mu+2}}$. If $k=0$ then we are done and if $k=1$ and $u^{0}=u_{1}$, then

$$
c=\partial_{f}\left(u^{0}\right)+\frac{N-\mu+2}{2(2 N-\mu)} S_{H, L}^{\frac{2 N-\mu}{N-\mu+2}}=\Upsilon_{f}(\Omega)+\frac{N-\mu+2}{2(2 N-\mu)} S_{H, L}^{\frac{2 N-\mu}{N-\mu+2}},
$$

a contradiction. If $k=1$ and $u^{0} \in \mathcal{N}_{f}^{-}$, we get

$$
c=\mathcal{J}_{f}\left(u^{0}\right)+\frac{N-\mu+2}{2(2 N-\mu)} S_{H, L}^{\frac{2 N-\mu}{N-\mu+2}} \geq \Upsilon_{f}^{-}(\Omega)+\frac{N-\mu+2}{2(2 N-\mu)} S_{H, L}^{\frac{2 N-\mu}{N-\mu+2}},
$$

which is again a contradiction. Hence $k=0$ and result follows.

\section{Existence of Second and third Solution}

In this section we will show the existence of second and third solution of problem $\left(P_{f}\right)$. To prove this, we shall show that for a sufficiently small $\delta>0$,

$$
\operatorname{cat}\left(\left\{u \in \mathcal{N}_{f}^{-}: \mathcal{J}_{f} \leq \Upsilon_{f}(\Omega)+\frac{N-\mu+2}{2(2 N-\mu)} S_{H, L}^{\frac{2 N-\mu}{N-2}}-\delta\right\}\right) \geq 2,
$$

where $\operatorname{cat}(X)$ is the category of the set $X$ is defined in the Definition 5.1. And then employing Lemma 5.2, we conclude the existence of second and third solutions. We shall first gather some preliminaries.

For $c \in \mathbb{R}$, we define

$$
b_{c}(u)=c b(u), \mathcal{J}_{c}(u)=\frac{1}{2} a(u)-\frac{1}{2.2_{\mu}^{\star}} b_{c}(u), \mathcal{M}_{c}=\left\{u \in H_{0}^{1}(\Omega) \backslash\{0\} \mid\left\langle\mathcal{J}_{c}^{\prime}(u), u\right\rangle=0\right\} .
$$

We denote

$$
\left[\mathcal{J}_{f} \leq c\right]=\left\{u \in \mathcal{N}_{f}^{-} \mid \mathcal{J}_{f}(u) \leq c\right\} .
$$

Definition 5.1. (i) For a topological space $X$, we say that a non-empty, closed subset $Y \subset X$ is contractible to a point in $X$ if and only if there exists a continuous mapping $\varrho:[0,1] \times Y \rightarrow X$ such that for some $x_{0} \in X$, $\varrho(0, x)=x$ for all $x \in Y$ and $\varrho(1, x)=x_{0}$ for all $x \in Y$. 
(ii) We define

$$
\begin{array}{r}
\operatorname{cat}(X)=\min \left\{k \in \mathbb{N} \mid \text { there exists closed subsets } Y_{1}, Y_{2}, \cdots Y_{k} \subset X\right. \text { such that } \\
\left.Y_{j} \text { is contractible to a point in } X \text { for all } j \text { and } \cup_{j=1}^{k} Y_{j}=X\right\}
\end{array}
$$

Lemma 5.2. [2] Suppose that $X$ is a Hilbert manifold and $G \in C^{1}(X, \mathbb{R})$. Assume that there are $c_{1} \in \mathbb{R}$ and $k \in \mathbb{N}$, such that

1. $\quad G$ satisfies the Palais-Smale condition for energy level $c \leq c_{1}$;

2. $\operatorname{cat}\left(\left\{x \in X \mid G(x) \leq c_{1}\right\}\right) \geq k$.

Then $G$ has at least $k$ critical points in $\left\{x \in X \mid G(x) \leq c_{1}\right\}$.

Lemma 5.3. [1, Theorem 2.5] Let $X$ be a topological space. Suppose that there are two continuous maps $\Phi$ : $\mathbb{S}^{N-1} \rightarrow X$ and $\Psi: X \rightarrow \mathbb{S}^{N-1}$ such that $\Psi \circ \Phi$ is homotopic to the identity map of $\mathbb{S}^{N-1}$. Then $\operatorname{cat}(X) \geq 2$.

Now we will proof a Lemma which will relate the functional $\mathcal{J}_{f}$ and $\mathcal{J}_{c}$. Note that for each $u \in H_{0}^{1}(\Omega)$ there exists a unique $t^{-}>0$ and a unique $t^{\star}>0$ such that $t^{-} u \in \mathcal{N}_{f}^{-}$and $t^{\star} u \in \mathcal{N}$.

Lemma 5.4. (i) For each $u \in \Sigma:=\left\{u \in H_{0}^{1}(\Omega) \mid\|u\|=1\right\}$, there exists a unique $t_{c}(u)>0$ such that $t_{c}(u) u \in \mathcal{M}_{c}$ and

$$
\max _{t \geq 0} \mathcal{J}_{c}(t u)=\mathcal{J}_{c}\left(t_{c}(u) u\right)=\frac{N-\mu+2}{2(2 N-\mu)}\left(b_{c}(u)\right)^{-\frac{N-2}{N-\mu+2}}
$$

(ii) For each $u \in H_{0}^{1}(\Omega)$ with $u^{+} \not \equiv 0$ and $0<\omega<1$, we have

$$
(1-\omega) \mathcal{J}_{\frac{1}{1-\omega}}(u)-\frac{1}{2 \omega}\|f\|_{H^{-1}}^{2} \leq \mathcal{J}_{f}(u) \leq(1+\omega) \mathcal{J}_{\frac{1}{1+\omega}}(u)+\frac{1}{2 \omega}\|f\|_{H^{-1}}^{2}
$$

(iii) For each $u \in \Sigma$ and $0<\omega<1$, we have

$$
(1-\omega)^{\frac{2 N-\mu}{N-\mu+2}} \mathcal{J}\left(t^{\star} u\right)-\frac{1}{2 \omega}\|f\|_{H^{-1}}^{2} \leq \partial_{f}\left(t^{-} u\right) \leq(1+\omega)^{\frac{2 N-\mu}{N-\mu+2}} \mathcal{J}\left(t^{\star} u\right)+\frac{1}{2 \omega}\|f\|_{H^{-1}}^{2} .
$$

(iv) There exists $e_{11}>0$ such that if $0<\|f\|_{H^{-1}}<e_{11}$ then $\Upsilon_{f}^{-}>0$.

\section{Proof.}

(i) For each $u \in \Sigma$, define $k(t)=\frac{1}{2} t^{2}-\frac{t^{2 \cdot 2_{\mu}^{\star}}}{2 \cdot 2_{\mu}^{\star}} b_{c}(u)$, then if

$$
t_{c}(u)=\left(\frac{1}{b_{c}(u)}\right)^{\frac{1}{2\left(2_{\mu}^{*}-1\right)}},
$$

we obtain $k^{\prime}\left(t_{c}(u)\right)=0$ and $k^{\prime \prime}\left(t_{c}(u)\right)<0$. Therefore, there exists a unique $t_{c}(u)>0$ such that

$$
\max _{t \geq 0} \mathcal{J}_{c}(t u)=\mathcal{J}_{c}\left(t_{c}(u) u\right)=\frac{N-\mu+2}{2(2 N-\mu)}\left(b_{c}(u)\right)^{-\frac{N-2}{N-\mu+2}} .
$$

(ii) For $0<\omega<1$, we have

$$
\left|\int_{\Omega} f u d x\right| \leq\|f\|_{H^{-1}}\|u\| \leq \frac{\omega}{2}\|u\|^{2}+\frac{1}{2 \omega}\|f\|_{H^{-1}}^{2},
$$

and for $u \in H_{0}^{1}(\Omega)$ with $u^{+} \not \equiv 0$ by the above inequality, we get

$$
\frac{1-\omega}{2}\|u\|^{2}-\frac{1}{2 \cdot 2_{\mu}^{\star}} b(u)-\frac{1}{2 \omega}\|f\|_{H^{-1}}^{2} \leq \mathcal{J}_{f}(u) \leq \frac{1+\omega}{2}\|u\|^{2}-\frac{1}{2.2_{\mu}^{\star}} b(u)+\frac{1}{2 \omega}\|f\|_{H^{-1}}^{2} .
$$

This implies that

$$
(1-\omega) \mathcal{J}_{\frac{1}{1-\omega}}(u)-\frac{1}{2 \omega}\|f\|_{H^{-1}}^{2} \leq \mathcal{J}_{f}(u) \leq(1+\omega) \mathcal{J}_{\frac{1}{1+\omega}}(u)+\frac{1}{2 \omega}\|f\|_{H^{-1}}^{2} .
$$


(iii) Using part (ii), we obtain the following estimate for each $u \in \Sigma$ and $0<\omega<1$

$$
(1-\omega) \mathcal{J}_{\frac{1}{1-\omega}}\left(t_{\frac{1}{1-\omega}}(u) u\right)-\frac{1}{2 \omega}\|f\|_{H^{-1}}^{2} \leq \mathcal{J}_{f}\left(t^{-}(u) u\right) \leq(1+\omega) \mathcal{J}_{\frac{1}{1+\omega}}\left(t_{\frac{1}{1+\omega}}(u) u\right)+\frac{1}{2 \omega}\|f\|_{H^{-1}}^{2} .
$$

Using (5.1) in part (i) we get

$$
\begin{aligned}
\mathcal{J}_{\frac{1}{1-\omega}}\left(t_{\frac{1}{1-\omega}}(u) u\right) & =\frac{N-\mu+2}{2(2 N-\mu)} b_{\frac{1}{1-\omega}}(u)^{-\frac{N-2}{N-\mu+2}} \\
& =(1-\omega)^{\frac{N-2}{N-\mu+2}} \frac{N-\mu+2}{2(2 N-\mu)} b(u)^{-\frac{N-2}{N-\mu+2}}=(1-\omega)^{\frac{N-2}{N-\mu+2}} \mathcal{J}\left(t^{\star} u\right) .
\end{aligned}
$$

Therefore, we get

$$
(1-\omega)^{\frac{2 N-\mu}{N-\mu+2}} \mathcal{J}\left(t^{\star} u\right)-\frac{1}{2 \omega}\|f\|_{H^{-1}}^{2} \leq \mathcal{J}_{f}\left(t^{-} u\right) \leq(1+\omega)^{\frac{2 N-\mu}{N-\mu+2}} \mathcal{J}\left(t^{*} u\right)-\frac{1}{2 \omega}\|f\|_{H^{-1}}^{2} .
$$

(iv) Combining part (iii) with the fact that $\Upsilon_{0}=\frac{N-\mu+2}{2(2 N-\mu)} S_{H, L}^{\frac{2 N-\mu}{N-\mu+2}}>0$ contributes that

$$
\begin{aligned}
\Upsilon_{f}^{-}(\Omega) & >(1-\omega)^{\frac{2 N-\mu}{N-\mu+2}} \Upsilon_{0}-\frac{1}{2 \omega}\|f\|_{H^{-1}}^{2} \\
& =(1-\omega)^{\frac{2 N-\mu}{N-\mu+2}} \frac{N-\mu+2}{2(2 N-\mu)} S_{H, L}^{\frac{2 N-\mu}{N-\mu+2}}-\frac{1}{2 \omega}\|f\|_{H^{-1}}^{2} .
\end{aligned}
$$

Thus, there exists $e_{11}>0$ such that $\Upsilon_{f}^{-}(\Omega)>0$ whenever $\|f\|_{H^{-1}}<e_{11}$

Lemma 5.5. If $\Omega$ satisfies condition $(A)$ then there exists a $\delta_{0}>0$ such that if $u \in \mathcal{N}$ with $\mathcal{J}(u) \leq \frac{N-\mu+2}{2(2 N-\mu)} S_{H, L}^{\frac{2 N-\mu}{N-\mu+2}}+$ $\delta_{0}$, then $\int_{\mathbb{R}^{N}} \frac{x}{|x|}|\nabla u|^{2} d x \neq 0$

Proof. Let $\left\{u_{n}\right\} \in \mathcal{N}$ such that $\mathcal{J}\left(u_{n}\right)=\frac{N-\mu+2}{2(2 N-\mu)} S_{H, L}^{\frac{2 N-\mu}{N-\mu+2}}+o(1)$ and $\int_{\mathbb{R}^{N}} \frac{x}{|x|}\left|\nabla u_{n}\right|^{2} d x=0$. Since $\left\{u_{n}\right\} \in \mathcal{N}$ therefore by Lemma 2.9, $\left\{u_{n}\right\}$ is a Palais-Smale sequence of $\mathcal{J}$ at level $\frac{N-\mu+2}{2(2 N-\mu)} S_{H, L}^{\frac{2 N-\mu}{N-\mu+2}}$. Now using [19, Theorem 4.4] and Remark 2.8, we have

$$
\left\|u_{n}-\left(\lambda_{n}^{1}\right)^{\frac{2-N}{2}} v_{1}\left(\left(.-y_{n}^{1}\right) / \lambda_{n}^{1}\right)\right\|_{D^{1,2}\left(\mathbb{R}^{N}\right)} \rightarrow 0,
$$

where $v_{1}$ is a minimizer of $S_{H, L}, \lambda_{n}^{1} \in \mathbb{R}^{+}, y_{n}^{1} \in \bar{\Omega}$. Moreover, if $n \rightarrow \infty$ then $\lambda_{n}^{1} \rightarrow 0, \frac{y_{n}^{1}}{\left|y_{n}^{1}\right|} \rightarrow y_{0}$ is the unit vector in $\mathbb{R}^{N}$. Thus we obtain

$$
\begin{aligned}
0=\int_{\mathbb{R}^{N}} \frac{x}{|x|}\left|\nabla u_{n}\right|^{2} d x & =\int_{\mathbb{R}^{N}} \frac{x}{|x|}\left(\left|\nabla u_{n}\right|^{2}-\left|\nabla\left(\lambda_{n}^{1}\right)^{\frac{2-N}{2}} v_{1}\left(\left(.-y_{n}^{1}\right) / \lambda_{n}^{1}\right)\right|^{2}\right) d x \\
& +\int_{\mathbb{R}^{N}} \frac{x}{|x|}\left|\nabla\left(\lambda_{n}^{1}\right)^{\frac{2-N}{2}} v_{1}\left(\left(.-y_{n}^{1}\right) / \lambda_{n}^{1}\right)\right|^{2} d x \\
& =o_{n}(1)+\int_{\mathbb{R}^{N}} \frac{y_{n}^{1}+\lambda_{n}^{1} z}{\left|y_{n}^{1}+\lambda_{n}^{1} z\right|}\left|\nabla v_{1}(z)\right|^{2} d z \\
& =o_{n}(1)+y_{0} S_{H, L}^{\frac{2 N-\mu}{N-\mu+2}},
\end{aligned}
$$

as $n \rightarrow \infty$, which is not possible.

For $0<\epsilon \leq \epsilon_{0}$ (defined in Proposition 4.5), define $H_{\epsilon}: \mathbb{S}^{N-1} \rightarrow H_{0}^{1}(\Omega)$ as

$$
H_{\epsilon}(\sigma)=u_{1}+s_{0} t_{0} g_{\rho}^{\epsilon, \sigma},
$$

where the function $u_{1}+s_{0} t_{0} g_{\rho}^{\epsilon, \sigma}$ defined in Lemma 4.6. 
Lemma 5.6. There exists a $\delta_{\epsilon} \in \mathbb{R}^{+}$such that

$$
H_{\epsilon}\left(\mathbb{S}^{N-1}\right) \subset\left[\partial_{f} \leq \Upsilon_{f}(\Omega)+\frac{N-\mu+2}{2(2 N-\mu)} S_{H, L}^{\frac{2 N-\mu}{N-\mu+2}}-\delta_{\epsilon}\right]
$$

Proof. Trivially, $H_{\epsilon}(\sigma)=u_{1}+s_{0} t_{0} g_{\rho}^{\epsilon, \sigma} \in \mathcal{N}_{f}^{-}$. So we only have to prove that $\partial_{f}\left(u_{1}+s_{0} t_{0} g_{\rho}^{\epsilon, \sigma}\right) \leq \Upsilon_{f}(\Omega)+$ $\frac{N-\mu+2}{2(2 N-\mu)} S_{H, L}^{\frac{2 N-\mu}{N-\mu+2}}-\delta_{\epsilon}$ for some $\delta_{\epsilon}>0$. Since by Proposition 4.5,

$$
\sup _{t \geq 0} \partial_{f}\left(u_{1}+t g_{\rho}^{\epsilon, \sigma}\right)<\partial_{f}\left(u_{1}\right)+\frac{N-\mu+2}{2(2 N-\mu)} S_{H, L}^{\frac{2 N-\mu}{N-\mu+2}}=\Upsilon_{f}(\Omega)+\frac{N-\mu+2}{2(2 N-\mu)} S_{H, L}^{\frac{2 N-\mu}{N-\mu+2}} \text {. }
$$

Hence there exists a $\delta_{\epsilon}>0$ such that

$$
\partial_{f}\left(u_{1}+s_{0} t_{0} g_{\rho}^{\epsilon, \sigma}\right) \leq \sup _{t \geq 0} \partial_{f}\left(u_{1}+\operatorname{tg}_{\rho}^{\epsilon, \sigma}\right) \leq \Upsilon_{f}(\Omega)+\frac{N-\mu+2}{2(2 N-\mu)} S_{H, L}^{\frac{2 N-\mu}{N-\mu+2}}-\delta_{\epsilon} .
$$

Lemma 5.7. There exists a $e_{22}>0$ such that $\|f\|_{H^{-1}}<e_{22}$ then for any

$$
u \in\left[\partial_{f} \leq \Upsilon_{f}(\Omega)+\frac{N-\mu+2}{2(2 N-\mu)} S_{H, L}^{\frac{2 N-\mu}{N-\mu+2}}\right] \text { we have } \int_{\mathbb{R}^{N}} \frac{x}{|x|}|\nabla u|^{2} d x \neq 0
$$

Proof. Let $u \in\left[\partial_{f} \leq \Upsilon_{f}(\Omega)+\frac{N-\mu+2}{2(2 N-\mu)} S_{H, L}^{\frac{2 N-\mu}{N-\mu+2}}\right]$ then $\mathcal{J}_{f}(u) \leq \Upsilon_{f}(\Omega)+\frac{N-\mu+2}{2(2 N-\mu)} S_{H, L}^{\frac{2 N-\mu}{N-\mu+2}}$ and $u \in \mathcal{N}_{f}^{-}$, that is, $\frac{1}{\|u\|} t^{-}\left(\frac{u}{\|u\|}\right)=1$. Since $\Upsilon_{f}(\Omega)<0$ we have $\partial_{f}(u) \leq \frac{N-\mu+2}{2(2 N-\mu)} S_{H, L}^{\frac{2 N-\mu}{N-\mu+2}}$. So for $\frac{u}{\|u\|} \in \Sigma$ there exits a $t^{\star}>0$ such that $\frac{t^{*} u}{\|u\|} \in \mathcal{N}$ which on using Lemma 5.4 (iii) implies

$$
(1-\omega)^{\frac{2 N-\mu}{N-\mu+2}} \mathcal{J}\left(t^{\star} \frac{u}{\|u\|}\right)-\frac{1}{2 \omega}\|f\|_{H^{-1}}^{2} \leq \mathcal{J}_{f}\left(t^{-} \frac{u}{\|u\|}\right)=\mathcal{J}_{f}(u) .
$$

Now using Lemma 3.4, we have

$$
\begin{aligned}
\mathcal{\partial}\left(t^{\star} \frac{u}{\|u\|}\right) \leq & (1-\omega)^{-\frac{2 N-\mu}{N-\mu+2}}\left(\partial_{f}(u)+\frac{1}{2 \omega}\|f\|_{H^{-1}}^{2}\right) \\
\leq & (1-\omega)^{-\frac{2 N-\mu}{N-\mu+2}}\left(\frac{N-\mu+2}{2(2 N-\mu)} S_{H, L}^{\frac{2 N-\mu}{N-\mu+2}}+\frac{1}{2 \omega}\|f\|_{H^{-1}}^{2}\right) \\
= & \left((1-\omega)^{-\frac{2 N-\mu}{N-\mu+2}}-1\right) \frac{N-\mu+2}{2(2 N-\mu)} S_{H, L}^{\frac{2 N-\mu}{N-\mu+2}} \\
& +\left(\frac{N-\mu+2}{2(2 N-\mu)} S_{H, L}^{\frac{2 N-\mu}{N-\mu+2}}+\frac{1}{2 \omega(1-\omega)^{\frac{2 N-\mu}{N-\mu+2}}}\|f\|_{H^{-1}}^{2}\right) .
\end{aligned}
$$

Choose $\omega_{0}>0$ such that for $0<\omega<\omega_{0}$, we have $\left((1-\omega)^{-\frac{2 N-\mu}{N-\mu+2}}-1\right) \frac{N-\mu+2}{2(2 N-\mu)} S_{H, L}^{\frac{2 N-\mu}{N-\mu+2}}<\frac{\delta_{0}}{2}$ where $\delta_{0}$ is defined in Lemma 5.5. Now for $0<\omega<\omega_{0}$ choose $e_{22}$ such that if $\|f\|_{H^{-1}}<e_{22}$ then $\frac{1}{2 \omega(1-\omega)^{\frac{2 N-\mu}{N-2}}}\|f\|_{H^{-1}}^{2}<\frac{\delta_{0}}{2}$. Therefore, we obtain

$$
\mathcal{J}\left(t^{\star} \frac{u}{\|u\|}\right) \leq \frac{N-\mu+2}{2(2 N-\mu)} S_{H, L}^{\frac{2 N-\mu}{N-\mu+2}}+\delta_{0}
$$

Using Lemma 5.5 we conclude the result. 
Define $G:\left[\mathcal{\partial}_{f} \leq \Upsilon_{f}(\Omega)+\frac{N-\mu+2}{2(2 N-\mu)} S_{H, L}^{\frac{2 N-\mu}{N-\mu+2}}\right] \rightarrow \mathbb{S}^{N-1}$ by

$$
G(u)=\frac{\int_{\mathbb{R}^{N}} \frac{x}{|x|}|\nabla u|^{2} d x}{\left.\left|\int_{\mathbb{R}^{N}} \frac{x}{|x|}\right| \nabla u\right|^{2} d x \mid} .
$$

Note that from Lemma 5.5, $G$ is well defined.

Lemma 5.8. For $0<\epsilon<\epsilon_{0}$ and $\|f\|_{H^{-1}}<e_{22}$, the map

$$
G \text { o } H_{\epsilon}: \mathbb{S}^{N-1} \rightarrow \mathbb{S}^{N-1}
$$

is homotopic to the identity.

Proof. Define $\mathcal{K}:=\left\{\left.u \in H_{0}^{1}(\Omega) \backslash\{0\}\left|\int_{\mathbb{R}^{N}} \frac{x}{|x|}\right| \nabla u\right|^{2} d x \neq 0\right\}$ and $\bar{G}: \mathcal{K} \rightarrow \mathbb{S}^{N-1}$ by

$$
\bar{G}(u)=\int_{\mathbb{R}^{N}} \frac{x}{|x|}|\nabla u|^{2} d x /\left.\left|\int_{\mathbb{R}^{N}} \frac{x}{|x|}\right| \nabla u\right|^{2} d x \mid
$$

as an extension of $G$. This on using Lemma 4.1 and Lemma 5.5, gives $\int_{\mathbb{R}^{N}} \frac{x}{|x|}\left|\nabla g_{\rho}^{\epsilon, \sigma}\right|^{2} d x \neq 0$ for sufficiently small $\epsilon$. Thus, $\bar{G}\left(g_{\rho}^{\epsilon, \sigma}\right)$ is well defined. Now let $y:\left[s_{1}, s_{2}\right] \rightarrow \mathbb{S}^{N-1}$ be a regular geodesic between $\bar{G}\left(g_{\rho}^{\epsilon, \sigma}\right)$ and $\bar{G}\left(H_{\epsilon}(\sigma)\right)$ such that $y\left(s_{1}\right)=\bar{G}\left(g_{\rho}^{\epsilon, \sigma}\right)$ and $y\left(s_{2}\right)=\bar{G}\left(H_{\epsilon}(\sigma)\right)$. Moreover, by a analogous argument as in Lemma 4.1, for $\delta_{0}>0$ there exists a $\epsilon_{0}>0$ such that

$$
\mathcal{J}\left(g_{\rho}^{2(1-\lambda) \epsilon}\right)<\frac{N-\mu+2}{2(2 N-\mu)} S_{H, L}^{\frac{2 N-\mu}{N-\mu+2}}+\delta_{0} \text { for all } 0<\epsilon<\epsilon_{0} \text { and } \sigma \in \mathbb{S}^{N-1}, \lambda \in\left[\frac{1}{2}, 1\right),
$$

where $\delta_{0}$ is defined in Lemma 5.5. Now define $\varsigma_{\epsilon}(\lambda, \sigma):[0,1] \times \mathbb{S}^{N-1} \rightarrow \mathbb{S}^{N-1}$ by

$$
\varsigma_{\epsilon}(\lambda, \sigma)= \begin{cases}y\left(2 \lambda\left(s_{1}-s_{2}\right)+s_{2}\right) & \text { if } \lambda \in\left[0, \frac{1}{2}\right), \\ \bar{G}\left(g_{\rho}^{2(1-\lambda) \epsilon}\right) & \text { if } \lambda \in\left[\frac{1}{2}, 1\right), \\ \sigma & \text { if } \lambda=1 .\end{cases}
$$

Clearly, $\varsigma_{\epsilon}$ is well defined. We claim that $\lim _{\lambda \rightarrow 1^{-}} \varsigma_{\epsilon}(\lambda, \sigma)=\sigma$ and $\lim _{\lambda \rightarrow \frac{1}{2}^{-}} \varsigma_{\epsilon}(\lambda, \sigma)=\bar{G}\left(g_{\rho}^{\epsilon, \sigma}\right)$.

(i) $\lim _{\lambda \rightarrow 1^{-}} \varsigma_{\epsilon}(\lambda, \sigma)=\sigma:$ Indeed

$$
\int_{\mathbb{R}^{N}} \frac{x}{|\chi|}\left|\nabla g_{\rho}^{2(1-\lambda) \epsilon}\right|^{2} d x=S_{H, L}^{\frac{2 N-\mu}{N-\mu+2}} \sigma+o(1) \text { as } \lambda \rightarrow 1^{-}
$$

then $\lim _{\lambda \rightarrow 1^{-}} \varsigma_{\epsilon}(\lambda, \sigma)=\sigma$.

(b) $\lim _{\lambda \rightarrow \frac{1}{2}^{-}} \varsigma_{\epsilon}(\lambda, \sigma)=\bar{G}\left(g_{\rho}^{\epsilon, \sigma}\right)$ : Indeed

$$
\lim _{\lambda \rightarrow \frac{1}{2}^{-}} \varsigma_{\epsilon}(\lambda, \sigma)=\lim _{\lambda \rightarrow \frac{1}{2}^{-}} y\left(2 \lambda\left(s_{1}-s_{2}\right)+s_{2}\right)=y\left(s_{1}\right)=\bar{G}\left(g_{\rho}^{\epsilon, \sigma}\right)
$$

Hence, $\varsigma_{\epsilon} \in C\left([0,1] \times \mathbb{S}^{N-1}, \mathbb{S}^{N-1}\right)$ and $\varsigma_{\epsilon}(0, \sigma)=\bar{G}\left(H_{\epsilon}(\sigma)\right)$ and $\varsigma_{\epsilon}(1, \sigma)=\sigma$ for $\sigma \in \mathbb{S}^{N-1}$ provided $0<\epsilon<\epsilon_{0}$ and $\|f\|_{H^{-1}}<e_{22}$. Thus the result follows. 
Proposition 5.9. Let $e^{\star}:=\min \left\{e_{00}, e_{11}, e_{22}\right\}$ where $e_{00} ; e_{11}$ and $e_{22}$ defined in Lemma 3.1, Lemma 5.4 and Lemma 5.7 respectively. Let $\|f\|_{H^{-1}}<e^{\star}$ then $\mathcal{J}_{f}$ has two critical points in

$$
\left[\partial_{f} \leq \Upsilon_{f}(\Omega)+\frac{N-\mu+2}{2(2 N-\mu)} S_{H, L}^{\frac{2 N-\mu}{N-\mu+2}}\right] .
$$

Equivalently, $\left(P_{f}\right)$ have another two different solutions which are different from $u_{1}$.

Proof. Using Lemma 5.8 and Lemma 5.3, we have

$$
\operatorname{cat}\left(\left[\partial_{f} \leq \Upsilon_{f}(\Omega)+\frac{N-\mu+2}{2(2 N-\mu)} S_{H, L}^{\frac{2 N-\mu}{N-\mu+2}}-\delta_{\epsilon}\right]\right) \geq 2 .
$$

Now the proof follows from Lemma 4.8(i) and Lemma 5.2.

\section{Existence of Fourth solution}

In this section we will prove the existence of high energy solution by using Brouwer's degree theory and minmax theorem given by Brezis and Nirenberg [8].

Let $\mathcal{V}:=\left\{u \in H_{0}^{1}(\Omega): \int_{\Omega} \int_{\Omega} \frac{\left|u^{+}(x)\right|^{2_{\mu}^{*}}\left|u^{+}(y)\right|^{2_{\mu}^{*}}}{|x-y|^{\mu}} d x d y=1\right\}, h_{\rho}^{\epsilon, \sigma}(x)=\frac{g_{\rho}^{\epsilon, \sigma}(x)}{\left\|g_{\rho}^{\epsilon, \sigma}\right\|_{N L}}$ where $g_{\rho}^{\epsilon, \sigma}$ is defined in (4.1).

Lemma 6.1. $\left\|h_{\rho}^{\epsilon, \sigma}\right\|_{D^{1,2\left(\mathbb{R}^{N}\right)}}^{2} \rightarrow S_{H, L}$ as $\epsilon \rightarrow 0$ uniformly in $\sigma \in \mathbb{S}^{N-1}$.

Proof. Proof follows from Lemma 4.1(i).

Lemma 6.2. There exists $a \rho_{0}>0$ such that for $0<\rho<\rho_{0}, \sup _{\sigma \in \mathbb{S}^{N-1}, \epsilon \in(0,1]}\left\|h_{\rho}^{\epsilon, \sigma}\right\|^{2}<2^{\frac{N-\mu+2}{2 N-\mu}} S_{H, L}$.

Proof. Since we know that $\left\|\nabla u_{\epsilon}^{\sigma}\right\|_{L^{2}\left(\mathbb{R}^{N}\right)}^{2}=\left\|u_{\epsilon}^{\sigma}\right\|_{N L}^{2.2_{\mu}^{*}}=S_{H, L}^{\frac{2 N-\mu}{N-\mu+2}}$ and this on using Lemma 4.2 we get $\sup _{\sigma \in \mathbb{S}^{N-1}, \epsilon \in(0,1]}\left\|h_{\rho}^{\epsilon, \sigma}\right\|^{2} \rightarrow S_{H, L}$ as $\rho \rightarrow 0$. So there exists a $\rho_{0}$ such that $0<\rho<\rho_{0}$, we obtain $\sup _{\sigma \in \mathbb{S}^{N-1}, \epsilon \in(0,1]}\left\|h_{\rho}^{\epsilon, \sigma}\right\|^{2}<2^{\frac{N-\mu+2}{2 N-\mu}} S_{H, L}$.

Now for any $u \in H_{0}^{1}(\Omega)$, by extending it to be zero outside $\Omega$, we define Barycenter mapping $\beta: \mathcal{V} \rightarrow \mathbb{R}^{N}$ as

$$
\beta(u)=\iint_{\mathbb{R}^{N}} \frac{x\left|u^{+}(x)\right|^{2_{\mu}^{*}}\left|u^{+}(y)\right|^{2_{\mu}^{*}}}{|x-y|^{\mu}} d x d y,
$$

and also let $Q:=\{u \in \mathcal{V}: \beta(u)=0\}$.

Lemma 6.3. There holds $\lim _{\epsilon \rightarrow 0} \beta\left(h_{\rho}^{\epsilon, \sigma}\right)=\sigma$. 
Proof. If there exists $\eta>0$ and a sequence $\epsilon_{n} \rightarrow 0^{+}$such that $\left|\beta\left(h_{\rho}^{\epsilon_{n}}\right)-\sigma\right| \geq \eta$. Then

$$
\begin{aligned}
& \beta\left(h_{\rho}^{\epsilon_{n}}\right)=\frac{\int_{\mathbb{R}^{N}} \int_{\mathbb{R}^{N}} \frac{x\left|h_{\rho}^{\epsilon_{n}}(x)\right|^{2_{\mu}^{*}}\left|h_{\rho}^{\epsilon_{n}}(y)\right|^{2_{\mu}^{*}}}{|x-y|^{\mu}} d x d y}{\left\|h_{\rho}^{\epsilon_{n}}\right\|_{N L}^{2.2_{\mu}^{*}}} \\
& =\sigma+\frac{\epsilon_{n} \iint_{\mathbb{R}^{N} \mathbb{R}^{N}} \frac{(z-\sigma)\left|v_{\rho}\left(\epsilon_{n} z+\left(1-\epsilon_{n}\right) \sigma\right)\right|^{2_{\mu}^{*}}\left|v_{\rho}\left(\epsilon_{n} w+\left(1-\epsilon_{n}\right) \sigma\right)\right|^{2_{\mu}^{*}}}{|z-w|{ }^{4}\left[1+|z|^{2}\right]^{\frac{2 N-\mu}{2}}\left[1+|w|^{2}\right]^{\frac{2 N-\mu}{2}}} d z d w}{\int_{\mathbb{R}^{N}} \int_{\mathbb{R}^{N}} \frac{\left|v_{\rho}\left(\epsilon_{n} z+\left(1-\epsilon_{n}\right) \sigma\right)\right|^{2_{\mu}^{*}}\left|\nu_{\rho}\left(\epsilon_{n} w+\left(1-\epsilon_{n}\right) \sigma\right)\right|^{2_{\mu}^{*}}}{|z-w| \mu\left[1+|z|^{2}\right]^{\frac{2 N-\mu}{2}}\left[1+|w|^{2}\right]^{\frac{2 N-\mu}{2}}} d z d w} \\
& \leq \sigma+\epsilon_{n} \sup _{z \in \operatorname{supp}\left(\nu_{\rho}\right)}|z-\sigma| \leq \sigma+C \epsilon_{n}, \text { for some } C>0 .
\end{aligned}
$$

It implies that $0<\eta \leq\left|\beta\left(h_{\rho}^{\epsilon_{n}}\right)-\sigma\right| \leq C \epsilon_{n} \rightarrow 0^{+}$as $\epsilon_{n} \rightarrow 0^{+}$, a contradiction.

Lemma 6.4. Let $m_{0}=\inf _{u \in \mathcal{Q}}\|u\|^{2}$ then $S_{H, L}<m_{0}$.

Proof. Obviously $S_{H, L} \leq m_{0}$, so let if possible, $S_{H, L}=\inf _{u \in \mathcal{Q}}\|u\|^{2}$ then there exists a sequence $\left\{v_{n}\right\} \in H_{0}^{1}(\Omega)$ such that $\left\|v_{n}\right\|_{N L}=1, \beta\left(v_{n}\right)=0,\left\|v_{n}\right\|^{2} \rightarrow S_{H, L}$ as $n \rightarrow \infty$. Setting $w_{n}=S_{H, L}^{\frac{N-2}{2(N-\mu+2)}} v_{n}$ we get $\left\|w_{n}\right\|_{N L}^{2.2_{\mu}^{*}}=S_{H, L}^{\frac{2 N-\mu}{N-\mu+2}}$ and $\left\|w_{n}\right\|^{2} \rightarrow S_{H, L}^{\frac{2 N-\mu}{N-\mu+2}}$. Therefore, $\mathcal{J}\left(w_{n}\right) \rightarrow \frac{N-\mu+2}{2(2 N-\mu)} S_{H, L}^{\frac{2 N-\mu}{N-\mu+2}}$ and $\mathcal{J}^{\prime}\left(w_{n}\right)\left(w_{n}\right)=o(1)$. Using Lemma 2.9, we obtain $\left\{w_{n}\right\}$ is a Palais-Smale sequence of $\mathcal{J}$ at level $\frac{N-\mu+2}{2(2 N-\mu)} S_{H, L}^{\frac{2 N-\mu}{N-2+2}}$. Subsequently, by [19, Theorem 4.4] and Remark 2.8, there exist sequences $y_{n} \in \Omega, \lambda_{n} \in \mathbb{R}^{+}$such that $y_{n} \rightarrow y_{0} \in \bar{\Omega}$ and $\lambda_{n} \rightarrow 0$, for the functions

$$
v_{n}=S_{H, L}^{-\frac{N-2}{2(N-\mu+2)}} w_{n} \text {, where } w_{n}=C\left(\frac{\lambda_{n}}{\lambda_{n}^{2}+\left|x-y_{n}\right|^{2}}\right)^{\frac{N-2}{2}} \text { for some } C>0 \text {. }
$$

Thus if

$$
\begin{aligned}
& C_{1}=C \int_{\mathbb{R}^{N}} \int_{\mathbb{R}^{N}} \frac{z}{|z-w|^{\mu}\left[1+|z|^{2}\right]^{\frac{2 N-\mu}{2}}\left[1+|w|^{2}\right]^{\frac{2 N-\mu}{2}}} d z d w \quad \text { and } \\
& C_{2}=C \int_{\mathbb{R}^{N}} \int_{\mathbb{R}^{N}} \frac{1}{|z-w|^{\mu}\left[1+|z|^{2}\right]^{\frac{2 N-\mu}{2}}\left[1+|w|^{2}\right]^{\frac{2 N-\mu}{2}}} d z d w,
\end{aligned}
$$

then

$$
0=\beta\left(v_{n}\right)=C \int_{\mathbb{R}^{N}} \int_{\mathbb{R}^{N}} \frac{x\left|v_{n}(x)\right|^{2_{\mu}^{*}}\left|v_{n}(y)\right|^{2_{\mu}^{*}}}{|x-y|^{\mu}} d x d y=\lambda_{n} C_{1}+y_{n} C_{2} \rightarrow C_{2} y_{0} .
$$

This is a contradiction. Hence $S_{H, L}<m_{0}$.

Lemma 6.5. There exists $\epsilon_{0}>0$ such that for $0<\epsilon<\epsilon_{0}$ and $|\sigma|=1$ we have

$$
S_{H, L}<\left\|h_{\rho}^{\epsilon, \sigma}\right\|_{D^{1,2\left(\mathbb{R}^{N}\right)}}^{2}<\frac{m_{0}+S_{H, L}}{2} .
$$

Proof. Apparently $S_{H, L} \leq\left\|h_{\rho}^{\epsilon, \sigma}\right\|_{D^{1,2}\left(\mathbb{R}^{N}\right)}^{2}$ and we know that $S_{H, L}$ is not attained on a bounded domain. Thus, $S_{H, L}<\left\|h_{\rho}^{\epsilon, \sigma}\right\|_{D^{1,2}\left(\mathbb{R}^{N}\right)}^{2}$. Since $S_{H, L}<m_{0}$, there exists $\delta_{0}$ such that $\frac{S_{H, L}}{2}+\delta_{0}<\frac{m_{0}}{2}$ and from Lemma 6.1 we know that $\left\|h_{\rho}^{\epsilon, \sigma}\right\|_{D^{1,2}\left(\mathbb{R}^{N}\right)}^{2} \rightarrow S_{H, L}$ as $\epsilon \rightarrow 0$. Therefore for $\delta_{0}>0$ there exists a $\epsilon_{0}>0$ such that $\left\|h_{\rho}^{\epsilon, \sigma}\right\|_{D^{1,2}\left(\mathbb{R}^{N}\right)}^{2}<$ $S_{H, L}+\delta_{0}$ whenever $0<\epsilon<\epsilon_{0}$. Hence we have the desired result. 
Now we will state the minimax lemma given by Brezis and Nirenberg [8].

Lemma 6.6. Let $Y$ be a Banach space and $\phi \in C^{1}(Y, \mathbb{R})$. Let $A$ be a compact metric space, $A_{0} \subset A$ be a closed set and $y \in C\left(A_{0}, Y\right)$. Define

$$
\Gamma=\left\{g \in C(A, Y): g(s)=y(s) \text { if } s \in A_{0}\right\}, \quad \bar{c}=\inf _{g \in \Gamma} \sup _{s \in A} \phi(g(s)), \quad \hat{c}=\sup _{y\left(A_{0}\right)} \phi .
$$

If $\bar{c}>\hat{c}$ then there exists a sequence $\left\{u_{n}\right\} \in Y$ satisfying $\phi\left(u_{n}\right) \rightarrow \bar{c}$ and $\phi^{\prime}\left(u_{n}\right) \rightarrow 0 .$. Further, if $\phi$ satisfies $(P S)_{\bar{c}}$ condition then there exists $u_{0} \in Y$ such that $\phi\left(u_{0}\right)=\bar{c}$ and $\phi^{\prime}\left(u_{0}\right)=0$.

Let $r_{0}=1-\epsilon_{0}$ and $\bar{B}_{r_{0}}=\left\{(1-\epsilon) \sigma \in \mathbb{R}^{N}:|(1-\epsilon) \sigma| \leq r_{0}, \sigma \in \mathbb{S}^{N-1}, 0<\epsilon \leq 1\right\}$, where $\epsilon_{0}$ is defined in Lemma 6.5. Then we set $F=\left\{q \in C\left(\bar{B}_{r_{0}}, \mathcal{V}\right) ; q_{\mid \partial \bar{B}_{r_{0}}}=h_{\rho}^{\epsilon, \sigma}\right\}$ and

$$
\bar{c}=\inf _{q \in F} \sup _{(1-\epsilon) \sigma \in \bar{B}_{r_{0}}}\|q((1-\epsilon) \sigma)\|^{2}, \quad \hat{c}=\sup _{\partial \bar{B}_{r_{0}}}\left\|h_{\rho}^{\epsilon, \sigma}\right\|^{2}
$$

Lemma 6.7. For each $q \in F$, we have $q\left(\bar{B}_{r_{0}}\right) \cap \mathcal{Q} \neq \emptyset$.

Proof. It is enough to show there exist $\tilde{e}>0$ and $\tilde{\sigma} \in \mathbb{S}^{N-1}$ such that $\beta(q((1-\tilde{e}) \tilde{\sigma}))=0$. Define $\psi: \bar{B}_{r_{0}} \rightarrow \mathbb{R}^{N}$ by $\psi((1-\epsilon) \sigma)=\beta(q((1-\epsilon) \sigma))$. We claim that

$$
d\left(\psi, \bar{B}_{r_{0}}, 0\right)=d\left(I, \bar{B}_{r_{0}}, 0\right) \neq 0 \text {, where } d \text { is Brouwer's topological degree. }
$$

If $(1-\epsilon) \sigma \in \partial \bar{B}_{r_{0}}$ then $q((1-\epsilon) \sigma)=h_{\rho}^{\epsilon, \sigma}$ which implies

$$
\psi((1-\epsilon) \sigma)=\beta(q((1-\epsilon) \sigma))=\beta\left(h_{\rho}^{\epsilon, \sigma}\right)=\sigma+o(1) \text { as } \epsilon \rightarrow 0 .
$$

Now define the homotopy $\mathcal{H}:[0,1] \times \bar{B}_{r_{0}} \rightarrow \mathbb{R}^{N}$ by

$$
\mathcal{H}(t,(1-\epsilon) \sigma)=(1-t) \psi((1-\epsilon) \sigma)+t I((1-\epsilon) \sigma)
$$

then for $(1-\epsilon) \sigma \in \partial \bar{B}_{r_{0}}$ and $t \in[0,1]$ we have

$$
\begin{aligned}
\mathcal{H}(t,(1-\epsilon) \sigma) & =(1-t) \sigma+o(1)+t\left(1-\epsilon_{0}\right) \sigma \\
& =o(1)+\left(1-\epsilon_{0} t\right) \sigma \neq 0, \quad \text { as } \epsilon \rightarrow 0 .
\end{aligned}
$$

So by Brouwer's degree theory, claim holds. It implies that there exist $\tilde{e}>0$ and $\tilde{\sigma} \in \mathbb{S}^{N-1}$ such that $\psi((1-\tilde{e}) \tilde{\sigma})=0$ that is, $\beta(q((1-\tilde{e}) \tilde{\sigma}))=0$.

Using above Lemma we have $m_{0} \leq \sup _{(1-\epsilon) \sigma \in \bar{B}_{r_{0}}}\|q((1-\epsilon) \sigma)\|^{2}$ for all $q \in F$. Hence

$$
m_{0} \leq \inf _{q \in F} \sup _{(1-\epsilon) \sigma \in \bar{B}_{r_{0}}}\|q((1-\epsilon) \sigma)\|^{2}=\bar{c} .
$$

Also, by the definition of $\bar{c}$, and Lemma 6.2, we have $\bar{c}<2^{\frac{N-\mu+2}{2 N-\mu}} S_{H, L}$ for $0<\rho<\rho_{0}$. Combining all these and using Lemma 6.4 we have

$$
S_{H, L}<m_{0} \leq \bar{c}<2^{\frac{N-\mu+2}{2 N-\mu}} S_{H, L} \text { for } \rho \text { sufficiently small . }
$$

In addition, from Lemma 6.5, we get

$$
\hat{c}=\sup _{\partial \bar{B}_{r_{0}}}\left\|h_{\rho}^{\epsilon, \sigma}\right\|^{2}<\frac{m_{0}+S_{H, L}}{2}<m_{0} \leq \bar{c} .
$$

Now we define

$$
\begin{aligned}
& \breve{\partial}_{f}(u)=\max _{t>0} \mathcal{J}_{f}(t u): \mathcal{V} \rightarrow \mathbb{R}^{N} \text { and } \breve{\partial}(u)=\max _{t>0} \mathcal{J}(t u): \mathcal{V} \rightarrow \mathbb{R}^{N}, \\
& y_{f}=\inf _{q \in F} \sup _{(1-\epsilon) \sigma \in \bar{B}_{r_{0}}} \breve{\partial}_{f}(q((1-\epsilon) \sigma)) \quad \text { and } \quad y_{0}=\inf _{q \in F} \sup _{(1-\epsilon) \sigma \in \bar{B}_{r_{0}}} \breve{\partial}(q((1-\epsilon) \sigma)) .
\end{aligned}
$$

We remark that the conclusion of Lemma 5.4 (iii) holds true for $\breve{\partial}_{f}$. Moreover, $\breve{\partial}_{f}(u)=\max _{t>0} \mathcal{\partial}_{f}(t u)=\mathcal{\partial}_{f}\left(t^{-}(u) u\right)$, where $t^{-}(u)$ is defined in Lemma 3.3. 
Lemma 6.8. The following holds:

(i) $\breve{\partial}_{f} \in C^{1}(\mathcal{V}, \mathbb{R})$ and $\left\langle\breve{\partial}_{f}^{\prime}(u), h\right\rangle=t^{-}(u)\left\langle\partial_{f}^{\prime}\left(t^{-}(u) u\right), h\right\rangle$ for all $h \in T_{u}(\mathcal{V})$ where

$$
T_{u}(\mathcal{V}):=\left\{h \in H_{0}^{1}(\Omega) \mid \int_{\Omega} \int_{\Omega} \frac{\left|u^{+}(x)\right|^{2_{\mu}^{*}}\left|u^{+}(y)\right|^{2_{\mu}^{*}-1} h(y)}{|x-y|^{\mu}}=0\right\} .
$$

(ii) If $u \in \mathcal{V}$ is a critical point of $\breve{\partial}_{f}$ then $t^{-}(u) u \in \mathcal{N}_{f}^{-}$is a critical point of $\mathcal{J}_{f}$.

(iii) If $\left\{u_{n}\right\}_{n \in \mathbb{N}}$ is a $(P S)_{c}$ sequence of $\breve{\partial}_{f}$ then $\left\{t^{-}\left(u_{n}\right) u_{n}\right\}_{n \in \mathbb{N}} \in \mathcal{N}_{f}^{-}$is a $(P S)_{c}$ sequence for $\mathfrak{J}_{f}$.

Proof. (i) For every $u \in H_{0}^{1}(\Omega), t^{-}(u) u \in \mathcal{N}_{f}^{-}$that is, $\left\langle\partial_{f}^{\prime}\left(t^{-}(u) u\right), u\right\rangle=0$ and $\left.\frac{d^{2}}{d t^{2}}\right|_{t=t^{-}(u)} \mathcal{J}_{f}(t u)<0$. Therefore, by implicit function theorem, we get $t^{-}(u) \in C^{1}(\mathcal{V},(0, \infty))$. As a result, $\breve{\partial}_{f}(u)=\mathfrak{J}_{f}\left(t^{-}(u) u\right) \in C^{1}(\mathcal{V}, \mathbb{R})$ and for all $h \in T_{u}(\mathcal{V})$, we have

$$
\left\langle\breve{g}_{f}^{\prime}(u), h\right\rangle=t^{-}(u)\left\langle\partial_{f}^{\prime}\left(t^{-}(u) u\right), h\right\rangle+\left\langle\mathcal{g}_{f}^{\prime}\left(t^{-}(u) u\right), u\right\rangle\left\langle\left(t^{-}(u)\right)^{\prime}, h\right\rangle=t^{-}(u)\left\langle\partial_{f}^{\prime}\left(t^{-}(u) u\right), h\right\rangle .
$$

(ii) Combining the fact that $u \in \mathcal{V}$ is a critical point of $\breve{\partial}_{f}$ and $\left\langle\partial_{f}^{\prime}\left(t^{-}(u) u\right), u\right\rangle=0$, we get the desired result. (iii) Let $\left\{u_{n}\right\}_{n \in \mathbb{N}}$ is a $(P S)_{c}$ sequence of $\breve{\partial}_{f}$, that is, $u_{n} \in \mathcal{V}, \breve{\partial}_{f}\left(u_{n}\right) \rightarrow c$ and

$$
\left\|\breve{\partial}_{f}^{\prime}(u)\right\|_{T_{u_{n}}^{*}(v)}=\sup \left\{\left|\left\langle\breve{g}_{f}^{\prime}\left(u_{n}\right), h\right\rangle\right|: h \in T_{u_{n}}(\mathcal{V}),\|h\|=1\right\} \rightarrow 0 \text { as } n \rightarrow \infty .
$$

By Lemma 3.3 we have $t^{-}\left(u_{n}\right)>\left(\frac{\|u\|^{2}}{2.22_{\mu}^{*}-1}\right)^{\frac{1}{2.2_{\mu}^{*}-2}}>\left(\frac{S_{H, L}}{2.2_{\mu}^{*}-1}\right)^{\frac{1}{2.2_{\mu^{*}-2}^{2}}}>C$ for some $C>0$. Since $H_{0}^{1}(\Omega)=R_{u_{n}} \oplus T_{u_{n}}(\mathcal{V})$ so $\left\langle\mathcal{J}_{f}^{\prime}\left(u_{n}\right), v\right\rangle=\left\langle\mathcal{J}_{f}^{\prime}\left(u_{n}\right), h_{v}\right\rangle$, where $h_{v}$ is the projection of $v$ in $T_{u_{n}}(\mathcal{V})$. Hence,

$$
\begin{aligned}
\left\|\partial_{f}^{\prime}\left(t^{-}\left(u_{n}\right) u_{n}\right)\right\| & =\sup _{v \in H_{0}^{1}(\Omega),\|v\|=1}\left|\left\langle\partial_{f}^{\prime}\left(t^{-}\left(u_{n}\right) u_{n}\right), v\right\rangle\right| \\
& =\sup _{v \in H_{0}^{1}(\Omega),\|v\|=1}\left|\left\langle\partial_{f}^{\prime}\left(t^{-}\left(u_{n}\right) u_{n}\right), h_{v}\right\rangle\right| \\
& =\sup _{v \in H_{0}^{1}(\Omega),\|v\|=1} \frac{1}{t^{-}\left(u_{n}\right)}\left|\left\langle\breve{\partial}_{f}^{\prime}\left(u_{n}\right), h_{v}\right\rangle\right| \leq \frac{1}{C}\left\|\breve{\partial}_{f}^{\prime}(u)\right\|_{T_{u_{n}}^{*}(v)} \rightarrow 0 .
\end{aligned}
$$

Clearly, $\partial_{f}\left(t^{-}\left(u_{n}\right) u_{n}\right) \rightarrow c$. Therefore, $\left\{t^{-}\left(u_{n}\right) u_{n}\right\}_{n \in \mathbb{N}} \in \mathcal{N}_{f}^{-}$is a $(P S)_{c}$ sequence for $\mathcal{J}_{f}$.

Lemma 6.9. If $0<\rho<\rho_{0}$, then $\frac{N-\mu+2}{2(2 N-\mu)} S_{H, L}^{\frac{2 N-\mu}{N-\mu+2}}<y_{0}<\frac{N-\mu+2}{2 N-\mu} S_{H, L}^{\frac{2 N-\mu}{N+2}}$.

Proof. For $u \in \mathcal{V}$, solving $f^{\prime}(t u)=t a(u)-t^{2.22_{\mu}^{*}-1}=0$ we get $t=0$ and $t=(a(u))^{\frac{1}{2.2_{\mu^{*}-2}}}$. Therefore,

$$
\breve{\partial}(u)=\max _{t>0} \mathcal{J}(t u)=\frac{N-\mu+2}{2(2 N-\mu)}\|u\|^{\frac{2(2 N-\mu)}{N-\mu+2}} .
$$

From the definition of $\bar{c}$, we obtain

$$
y_{0}=\frac{N-\mu+2}{2(2 N-\mu)} \inf _{q \in F} \sup _{(1-\epsilon) \sigma \in \bar{B}_{r_{0}}}\|q((1-\epsilon) \sigma)\|^{\frac{2(2 N-\mu)}{N-\mu+2}}=\frac{N-\mu+2}{2(2 N-\mu)} \bar{c}^{\frac{2 N-\mu}{N-\mu+2}}
$$

which on using (6.1) yields the desired result.

Lemma 6.10. $\breve{\partial}_{f}\left(h_{\rho}^{\epsilon, \sigma}\right)=\frac{N-\mu+2}{2(2 N-\mu)} S_{H, L}^{\frac{2 N-\mu}{N-\mu+2}}+o(1)$ as $\epsilon \rightarrow 0$.

Proof. By Lemma 4.1, $h_{\rho}^{\epsilon, \sigma} \rightarrow 0$ in $H_{0}^{1}(\Omega)$ as $\epsilon \rightarrow 0$. On solving

$$
\partial_{f}^{\prime}\left(t h_{\rho}^{\epsilon, \sigma}\right)=t a\left(h_{\rho}^{\epsilon, \sigma}\right)-t^{2.2_{\mu}^{\star}-1}-\int_{\Omega} f h_{\rho}^{\epsilon, \sigma} d x=0,
$$

we conclude $t_{f}=\left\|g_{\rho}^{\epsilon, \sigma}\right\|_{N L}+o(1)$. Hence again from the Lemma 4.1 we obtain $\breve{\partial}_{f}\left(h_{\rho}^{\epsilon, \sigma}\right)=\max _{t>0} \mathcal{J}_{f}\left(t h_{\rho}^{\epsilon, \sigma}\right)=\mathcal{J}_{f}\left(t_{f} h_{\rho}^{\epsilon, \sigma}\right)=\mathcal{J}_{f}\left(g_{\rho}^{\epsilon, \sigma}\right)=\frac{N-\mu+2}{2(2 N-\mu)} S_{H, L}^{\frac{2 N-\mu}{N-\mu+2}}+o(1)$ as $\epsilon \rightarrow 0$. 
Lemma 6.11. There exists $e_{0}^{\star}>0$ such that if $0<\|f\|_{H^{-1}}<e_{0}^{\star}$,

$$
\Upsilon_{f}(\Omega)+\frac{N-\mu+2}{2(2 N-\mu)} S_{H, L}^{\frac{2 N-\mu}{N-\mu+2}}<y_{f}<\Upsilon_{f}^{-}(\Omega)+\frac{N-\mu+2}{2(2 N-\mu)} S_{H, L}^{\frac{2 N-\mu}{N-\mu+2}} .
$$

Proof. Analogous to the proof of Lemma 5.4(iii) we can have

$$
(1-\omega)^{\frac{2 N-\mu}{N-\mu+2}} \mathcal{J}\left(t^{\star} u\right)-\frac{1}{2 \omega}\|f\|_{H^{-1}}^{2} \leq \mathcal{J}_{f}\left(t^{-} u\right) \leq(1+\omega)^{\frac{2 N-\mu}{N-\mu+2}} \mathcal{J}\left(t^{\star} u\right)+\frac{1}{2 \omega}\|f\|_{H^{-1}}^{2} .
$$

Using the above inequality with the definition of $\breve{\partial}$ and $\breve{g}_{f}$, we get

$$
(1-\omega)^{\frac{2 N-\mu}{N-\mu+2}} \breve{\partial}(u)-\frac{1}{2 \omega}\|f\|_{H^{-1}}^{2} \leq \breve{\partial ̆}_{f}(u) \leq(1+\omega)^{\frac{2 N-\mu}{N-\mu+2}} \breve{\partial}(u)+\frac{1}{2 \omega}\|f\|_{H^{-1}}^{2} .
$$

For $\delta>0$ there exists $e_{1}(\delta)$ such that if $\|f\|_{H^{-1}}<e_{1}(\delta)$ then

$$
y_{0}-\delta<y_{f}<y_{0}+\delta .
$$

Now from Lemma 5.4(iii) for each $0<\omega<1$, we have

$$
\begin{aligned}
& (1-\omega)^{\frac{2 N-\mu}{N-\mu+2}} \frac{N-\mu+2}{2(2 N-\mu)} S_{H, L}^{\frac{2 N-\mu}{N-2+2}}-\frac{1}{2 \omega}\|f\|_{H^{-1}}^{2} \\
& \quad \leq \Upsilon_{f}^{-}(\Omega) \leq(1+\omega)^{\frac{2 N-\mu}{N-\mu+2}} \frac{N-\mu+2}{2(2 N-\mu)} S_{H, L}^{\frac{2 N-\mu}{N-\mu+2}}+\frac{1}{2 \omega}\|f\|_{H^{-1}}^{2} .
\end{aligned}
$$

So for $\delta>0$ there exists $e_{2}(\delta)>0$ such that whenever $\|f\|_{H^{-1}}<e_{2}(\delta)$ then

$$
\frac{N-\mu+2}{2(2 N-\mu)} S_{H, L}^{\frac{2 N-\mu}{N-\mu+2}}-\delta \leq \Upsilon_{f}^{-}(\Omega) \leq \frac{N-\mu+2}{2(2 N-\mu)} S_{H, L}^{\frac{2 N-\mu}{N-\mu+2}}+\delta .
$$

It implies

$$
\frac{N-\mu+2}{2 N-\mu} S_{H, L}^{\frac{2 N-\mu}{N-\mu+2}}-\delta \leq \Upsilon_{f}^{-}(\Omega)+\frac{N-\mu+2}{2(2 N-\mu)} S_{H, L}^{\frac{2 N-\mu}{N-\mu+2}} \leq \frac{N-\mu+2}{2 N-\mu} S_{H, L}^{\frac{2 N-\mu}{N-\mu+2}}+\delta .
$$

Moreover, from Lemma 6.9

$$
\frac{N-\mu+2}{2(2 N-\mu)} S_{H, L}^{\frac{2 N-\mu}{N-\mu+2}}<y_{0}<\frac{N-\mu+2}{2 N-\mu} S_{H, L}^{\frac{2 N-\mu}{N-\mu+2}}
$$

Hence for fix small $0<\epsilon<\min \left\{\frac{\frac{N-\mu+2}{2 N-\mu} S_{H, L}^{\frac{2 N-\mu}{N-\mu}-y_{0}}}{2}, y_{0}-\frac{N-\mu+2}{2(2 N-\mu)} S_{H, L}^{\frac{2 N-\mu}{N-\mu+2}}\right\}$ such that if $\|f\|_{H^{-1}}<e_{0}^{\star}=$ $\min \left\{e_{2}(\epsilon), e_{2}(\epsilon)\right\}$ then using (6.2) and (6.3), we obtain

$$
\begin{aligned}
& \Upsilon_{f}(\Omega)+\frac{N-\mu+2}{2(2 N-\mu)} S_{H, L}^{\frac{2 N-\mu}{N-\mu+2}}<\frac{N-\mu+2}{2(2 N-\mu)} S_{H, L}^{\frac{2 N-\mu}{N-\mu+2}}<y_{0}-\epsilon \leq y_{f} \quad \text { and } \\
& y_{f}<y_{0}+2 \epsilon-\epsilon<\frac{N-\mu+2}{2 N-\mu} S_{H, L}^{\frac{2 N-\mu}{N-\mu+2}}-\epsilon \leq \Upsilon_{f}^{-}(\Omega)+\frac{N-\mu+2}{2(2 N-\mu)} S_{H, L}^{\frac{2 N-\mu}{N-\mu+2}} .
\end{aligned}
$$

That is, $\Upsilon_{f}(\Omega)+\frac{N-\mu+2}{2(2 N-\mu)} S_{H, L}^{\frac{2 N-\mu}{N-\mu+2}}<y_{f}<\Upsilon_{f}^{-}(\Omega)+\frac{N-\mu+2}{2(2 N-\mu)} S_{H, L}^{\frac{2 N-\mu}{N-\mu+2}}$.

Proposition 6.12. If $0<\rho<\rho_{0}, 0<\|f\|_{H^{-1}}<e_{0}^{\star}$ (defined in Lemma 6.11) then there exists a critical point $u_{4} \in \mathcal{N}_{f}^{-}$of $\partial_{f}$ with $\partial_{f}\left(u_{4}\right)=y_{f}$.

Proof. Let $c \in\left(\Upsilon_{f}(\Omega)+\frac{N-\mu+2}{2(2 N-\mu)} S_{H, L}^{\frac{2 N-\mu}{N-\mu+2}}, \Upsilon_{f}^{-}(\Omega)+\frac{N-\mu+2}{2(2 N-\mu)} S_{H, L}^{\frac{2 N-\mu}{N-\mu+2}}\right)$ and $\left\{u_{n}\right\}_{n \in \mathbb{N}}$ is a $(P S)_{c}$ sequence of $\breve{\partial}_{f}$. Then by Lemma 6.8, $\left\{t^{-}\left(u_{n}\right) u_{n}\right\}_{n \in \mathbb{N}} \in \mathcal{N}_{f}^{-}$is a $(P S)_{c}$ sequence for $\mathcal{J}_{f}$ which on using Lemma 4.8 gives that $\left\{u_{n}\right\}_{n \in \mathbb{N}}$ is compact. Moreover, from Lemma 6.10, $y_{f}>\breve{\partial}_{f}\left(h_{\rho}^{\epsilon, \sigma}\right)=\frac{N-\mu+2}{2(2 N-\mu)} S_{H, L}^{\frac{2 N-\mu}{N-\mu+2}}+o(1)$ as $\epsilon$ sufficiently small. Using Lemma 6.6 we have $y_{f}$ is a critical value of $\breve{\partial}_{f}$. Therefore, there exists $v_{4} \in \mathcal{V}$ such that $\breve{\partial}_{f}\left(v_{4}\right)=y_{f}$ and $\breve{g}_{f}^{\prime}\left(v_{4}\right)=0$. Thus by Lemma 6.8, $u_{4}:=t^{-}\left(v_{4}\right) v_{4} \in \mathcal{N}_{f}^{-}$is a critical point of $\mathcal{J}_{f}$ and $\mathcal{J}_{f}\left(u_{4}\right)=y_{f}$. 
Proof of Theorem 1.1 : First note that by Lemma 3.8, we have all solutions of $\left(P_{f}\right)$ are positive in $\Omega$ and from Lemma 3.7, we have $u_{1} \in \mathcal{N}_{f}^{+} \subset H_{0}^{1}(\Omega)$ such that $\mathcal{J}_{f}\left(u_{1}\right)=\Upsilon_{f}$ whenever $0<\|f\|_{H^{-1}}<e_{00}$. By Proposition 5.9 we have two more critical point $u_{2}, u_{3} \in \mathcal{N}_{f}^{-}$of $\partial_{f}$ such that in $\partial_{f}\left(u_{2}\right), \partial_{f}\left(u_{3}\right)<\Upsilon_{f}(\Omega)+\frac{N-\mu+2}{2(2 N-\mu)} S_{H, L}^{\frac{2 N-\mu}{N-\mu+2}}$. Therefore we get three positive solutions of $\left(P_{f}\right)$ whenever $0<\|f\|_{H^{-1}}<e^{\star}$ where $e^{\star}$ is defined in Proposition 5.9. Let $e^{\star \star}=\min \left\{e^{\star}, e_{0}^{\star}\right\}$ then by Proposition 6.12, we get $u_{4} \in \mathcal{N}_{f}^{-} \partial_{f}\left(u_{4}\right)=y_{f}$.

\section{References}

[1] S. Adachi and K. Tanaka, Four positive solutions for the semilinear elliptic equation: $-\Delta u+u=a(x) u^{p}+f(x)$ in $\mathbb{R}^{N}$, Calc. Var. Partial Differential Equations 11 (2000), no. 1, 63-95.

[2] A. Ambrosetti, Critical points and nonlinear variational problems, Mém. Soc. Math. France (N.S.) 49 (1992), 1-139.

[3] A. Bahri and J. M. Coron, On a nonlinear elliptic equation involving the critical Sobolev exponent: the effect of the topology of the domain, Comm. Pure Appl. Math. 41 (1988), no. 3, 253-294.

[4] V. Benci and G. Cerami, The effect of the domain topology on the number of positive solutions of nonlinear elliptic problems, Arch. Rational Mech. Anal. 114 (1991), no. 1, 79-93.

[5] V. Benci, G. Cerami and D. Passaseo, On the number of the positive solutions of some nonlinear elliptic problems, in “ Nonlinear analysis, Sc. Norm. Super. di Pisa Quaderni, Scuola Norm. Sup., Pisa (1991), 93-107,

[6] V. Benci and G. Cerami, Multiple positive solutions of some elliptic problems via the Morse theory and the domain topology, Calc. Var. Partial Differential Equations 2 (1994), no. 1, 29-48.

[7] H. Brezis and L. Nirenberg, A minimization problem with critical exponent and nonzero data, in "Symmetry in Nature", Scuola Norm. Sup. Pisa 1 (1989), 129-140.

[8] H. Brezis and L. Nirenberg, Remarks on finding critical points, Comm. Pure Appl. Math. 44 (1991), no. 8-9, 939-963.

[9] D.M. Cao and H.S. Zhou, Multiple positive solutions of nonhomogeneous semilinear elliptic equations in $\mathbb{R}^{N}$, Proc. Roy. Soc. Edinburgh Sect. A 126 (1996), no. 2, 443-463.

[10] M. Clapp, M. Del Pino and M. Musso, Multiple solutions for a non-homogeneous elliptic equation at the critical exponent, Proc. Roy. Soc. Edinburgh Sect. A 134 (2004), no. 1, 69-87.

[11] M. Clapp, O. Kavian and B. Ruf, Multiple solutions of nonhomogeneous elliptic equations with critical nonlinearity on symmetric domains, Commun. Contemp. Math. 5 (2003), no. 2, 147-169.

[12] J.M. Coron, Topologie et cas limite des injections de Sobolev,C. R. Acad. Sci. Paris Sèr. I Math. 299 (1984), no. 7, 209-12.

[13] E.N. Dancer, A note on an equation with critical exponent, Bull. London Math. Soc. 20 (1988), 600-602.

[14] F. Gao, and M. Yang, On nonlocal Choquard equations with Hardy-Littlewood-Sobolev critical exponents, J. Math. Anal. Appl. 448 (2017), no. 2, 1006-1041.

[15] F. Gao and M. Yang, On the Brezis-Nirenberg type critical problem for nonlinear Choquard equation, Sci. China Math. 61 (2018), no. 7, 1219-1242.

[16] M. Ghimenti and J. Van Schaftingen, Nodal solutions for the Choquard equation, J. Functional Analysis 271 (2016), no. 1, 107-135.

[17] J. Giacomoni, T. Mukherjee and K. Sreenadh, Doubly nonlocal system with Hardy-Littlewood-Sobolev critical nonlinearity, $J$. Math. Anal. Appl. 467 (2018), no. 1, 638-672.

[18] D. Goel and K. Sreenadh, Kirchhoff equations with Hardy-Littlewood-Sobolev critical nonlinearity, Nonlinear Anal. https://doi.org/10.1016/j.na.2019.01.035.

[19] D. Goel, V. D. Rădulescu and K. Sreenadh, Coron problem for nonlocal equations invloving Choquard nonlinearity, arXiv:1804.08084 (2018).

[20] H. He and J. Yang, Positive solutions for critical inhomogeneous elliptic problems in non-contractible domains, Nonlinear Anal. 70 (2009), no. 2, 952-973.

[21] N. Hirano, Multiple existence of solutions for a nonhomogeneous elliptic problem on $\mathbb{R}^{N}$, J. Math. Anal. Appl. 336 (2007), no. 1, 506-522.

[22] E.H. Lieb, Existence and uniqueness of the minimizing solution of Choquard's nonlinear equation, Studies in Appl. Math. 57 (1976/77), no. 2, 93-105.

[23] E.H. Lieb, M. Loss, Analysis, Second edition, Graduate Studies in Mathematics, American Mathematical Society, Providence, RI, 2001.

[24] V. Moroz and J. Van Schaftingen, Semi-classical states for the Choquard equation, Calc. Var. Partial Differential Equations 52 (2015), no. 1-2, 199-235.

[25] V. Moroz and J. Van Schaftingen, Existence of groundstates for a class of nonlinear Choquard equations, Trans. Amer. Math. Soc. 367 (2015), 6557-6579. 
[26] V. Moroz, J. Van Schaftingen, Groundstates of nonlinear Choquard equations: Hardy-Littlewood-Sobolev critical exponent, Commun. Contemp. Math. 17 (2015), no. 5, 1550005.

[27] V. Moroz and J. Van Schaftingen, Groundstates of nonlinear Choquard equations: existence, qualitative properties and decay asymptotics, J. Functional Analysis 265 (2013), no. 2, 153-184.

[28] S. Pekar, Untersuchung über die Elektronentheorie der Kristalle, Akademie Verlag, Berlin, 1954.

[29] O. Rey, A multiplicity result for a variational problem with lack of compactness, Nonlinear Anal. 13 (1989), no. 10, 12411249.

[30] Z. Shen, F. Gao and M. Yang, Multiple solutions for nonhomogeneous Choquard equation involving Hardy-LittlewoodSobolev critical exponent, Z. Angew. Math. Phys. 68 (2017), no. 3, 61.

[31] G. Tarantello, On nonhomogeneous elliptic equations involving critical Sobolev exponent, Ann. Inst. H. Poincarè Anal. Non Linèaire 9 (1992), no. 3, 281-304.

[32] T.F. Wu, Three positive solutions for Dirichlet problems involving critical Sobolev exponent and sign-changing weight, $J$. Differential Equations 249 (2010), no. 7, 1549-1578.

[33] T.F. Wu, Multiple positive solutions of non-homogeneous elliptic equations in exterior domains, Proc. Roy. Soc. Edinburgh Sect. A 137 (2007), no. 3, 603-624. 